\title{
Bifurcation Analysis and Sliding Mode Control of Chaotic Vibrations in an Autonomous System
}

\author{
Wenju Du, Yandong Chu, Jiangang Zhang, Yingxiang Chang, Jianning Yu, and Xinlei An \\ Department of Mathematics, Lanzhou Jiaotong University, Lanzhou, Gansu 730070, China \\ Correspondence should be addressed to Jiangang Zhang; zhangjg7715776@126.com
}

Received 13 March 2014; Revised 23 June 2014; Accepted 24 June 2014; Published 6 August 2014

Academic Editor: Chao Yan

Copyright ( 2014 Wenju Du et al. This is an open access article distributed under the Creative Commons Attribution License, which permits unrestricted use, distribution, and reproduction in any medium, provided the original work is properly cited.

\begin{abstract}
We study the bifurcations and sliding mode control of chaotic vibrations in an autonomous system. More precisely, a Hopf bifurcation controller is designed so as to control the unstable subcritical Hopf bifurcation to the stable supercritical Hopf bifurcation. Research result shows that the control method can work very well in Hopf bifurcation control. Besides, we controlled the system to any fixed point and any periodic orbit to eliminate the chaotic vibration by means of sliding mode method. And the numerical simulations were presented to confirm the effectiveness of the controller.
\end{abstract}

\section{Introduction}

In 1963, Lorenz discovered chaos in a simple system of three autonomous ordinary differential equations in order to describe the simplified Rayleigh-Benard problem [1]. From then on, some other chaotic systems were established, such as Chen system [2], Lü system [3], and Chu system [4]. Despite the simplicity of three-dimensional autonomous systems, these systems have a rich dynamical behavior, ranging from stable equilibrium points to periodic and even chaotic oscillations, depending on the parameter values. Moreover, bifurcation analysis and numerical simulation for these systems have been done by many researchers, such as [5-14]. In 2008, a new three-dimensional Lorenz-like chaotic system is reported; nonlinear characteristic and basic dynamic properties of the three-dimensional autonomous system are studied by means of nonlinear dynamics theory, including the stability and the conditions for generating Hopf bifurcation of the equilibria [15]. Sotomayor et al. [16] use the projection method described in [17] for the calculation of the first and second Lyapunov coefficients associated with Hopf bifurcations of the Watt governor system, and it was extended to the calculation of the third and fourth Lyapunov coefficients. Dias et al. [18] studied the existence of singularly degenerate heteroclinic cycles for a suitable choice of the parameters in a Lorenz-like system. Zhang et al. [19] reported the finding of a new simple three-dimensional quadratic chaotic system with three quadratic nonlinearities obtained by adding a cross-product nonlinear term to the second equation of the Rucklidge system.

In recent years, the research of robust control system made great progress not only in theory but also in practical application. As a representative of the nonlinear robust control theory, variable structure control theory has been widely researched throughout the world and also had an increasing number of industrial applications. Wang et al. [20] present two methods to design a single-input/single-output integral variable structure system. Lee et al. [21] present a sliding mode controller with integral compensation for a magnetic suspension balance beam system. The control scheme comprises an integral controller which is designed for achieving zero steady-state error under step disturbances and a sliding mode controller which is designed for enhancing robustness under plant uncertainties. Di-Yi et al. [22] proposed a no-chattering sliding mode control strategy for a class of fractional-order chaotic systems. The designed control scheme guarantees the asymptotical stability of an uncertain fractional-order chaotic system. To ensure the robustness of the system control, Chen et al. [23] stabilized the chaotic orbits to arbitrary chosen fixed points and periodic orbits by means of sliding mode method, and MATLAB simulations were presented to confirm the validity of the controller. 
Chen et al. [24] studied the nonlinear dynamics behavior of hydroturbine governing system. In order to eliminate the chaotic vibration, the author used sliding mode method and MATLAB simulations and controlled the system to any fixed point and any periodic orbit. The results show that using sliding mode method can make the system track target orbit strictly and smoothly with short transition time. In this paper, we mainly consider a new chaotic system proposed by Gao [25]. Gao constructed a three-dimensional continuous autonomous chaotic system, which is different from classic Lorenz system, Chen system, and Lü system. Some basic dynamical properties of the new system are analyzed by means of theoretical analysis and nonlinear techniques, such as phase diagram, time response diagram, Lyapunov exponent spectrum, and bifurcation diagram. However, the relationship between the Hopf bifurcation and the system parameters has not been clarified yet. And there is not any analysis of chaos control.

The main purpose of this paper is to investigate the bifurcations and sliding mode control of chaotic vibrations in an autonomous system (1). The rest of this paper is organized as follows. In Section 2, we present the linear analysis of equilibrium of system (1). A brief review of the method used to study codimensions one and two Hopf bifurcations is presented in Section 3. The direction of Hopf bifurcation and the stability of bifurcating periodic solutions are analyzed in detail in Section 4. In Section 5, we designed a controller such that the equilibrium $E_{+}$undergoes a controllable Hopf bifurcation. In Section 6, nonlinear dynamic properties of this chaotic system are studied by means of bifurcation diagrams. We controlled the system to any fixed point and any periodic orbit to eliminate the chaotic vibration by means of sliding mode method in Section 7. Section 8 concludes the paper.

\section{Linear Analysis of System (1)}

In this paper, we investigate Hopf bifurcation of a new chaotic of the form [25]

$$
\begin{aligned}
& \dot{x}=y z+a(x-y), \\
& \dot{y}=x-y, \\
& \dot{z}=b-x y,
\end{aligned}
$$

where $(x, y, z) \in \mathbf{R}^{3}$ are the state variables and $(a, b) \in \mathbf{R}^{2}$ are real parameters. In this section, we study some of the generalities and linear stability of system (1). In a vectorial notation which will be useful in the calculations, system (1) can be written as $x^{\prime}=f(x, \zeta)$, where

$$
f(x, \zeta)=(y z+a(x-y), x-y, b-x y),
$$

$x=(x, y, z) \in \mathbf{R}^{3}$ and $\zeta=(a, b) \in \mathbf{R}^{2}$.

The equilibria of system (1) can be found by solving the following equations simultaneously:

$$
y z+a(x-y)=0, \quad x-y=0, \quad b-x y=0 .
$$

From (3), the system (1) has two equilibria $E_{ \pm}=( \pm \sqrt{b}, \pm \sqrt{b}$, $0)$ if $b>0$.

Because the system is invariant under the transformation $S:(x, y, z) \rightarrow(-x,-y, z)$, stability of $E_{+}$and $E_{-}$can be calculated similarly, so, one only needs to consider the stability of any one of them. The stability of the system at the fixed point $E_{+}$is analyzed.

Proposition 1. If $a>a_{0}$, the equilibrium $E_{+}$is always unstable. If $b(1-a)>2 b$ and

$$
a<a_{0}=-1
$$

then the equilibrium $E_{+}$is asymptotically stable.

Proof. At the fixed point $E_{+}=(\sqrt{b}, \sqrt{b}, 0)$, the Jacobian matrix is defined as

$$
\mathbf{A}_{+}=\left(\begin{array}{ccc}
a & z-a & y \\
1 & -1 & 0 \\
-y & -x & 0
\end{array}\right)_{(\sqrt{b}, \sqrt{b}, 0)}=\left(\begin{array}{ccc}
a & -a & \sqrt{b} \\
1 & -1 & 0 \\
-\sqrt{b} & -\sqrt{b} & 0
\end{array}\right) .
$$

The characteristic polynomial of the Jacobian matrix of system (1) at $E_{+}$has the form

$$
p(\lambda)=\lambda^{3}+(1-a) \lambda^{2}+b \lambda+2 b .
$$

If $a>a_{0}$, according to Routh-Hurwitz criterion, the equilibrium $E_{+}$is unstable. According to Routh-Hurwitz criterion, the real parts of all the roots $\lambda$ of (6) are negative if and only if

$$
1-a>0, \quad b(1-a)>2 b,
$$

so the proposition follows.

The equation $a=a_{0}$ in (4) gives the equation of the Hopf curvein the parameter plane $(a, b)$. This equation will be used in Section 4 in the study of Hopf bifurcations which occur at the equilibria $E_{+}$of system (1).

\section{Outline of the Hopf Bifurcation Methods}

This section is a review of the projection method described in [15-18] for the calculation of the first Lyapunov coefficients and second Lyapunov coefficients associated with Hopf bifurcation, denoted by $l_{1}$ and $l_{2}$, respectively.

Consider the differential equation

$$
x^{\prime}=f(x, \zeta),
$$

where $x \in \mathbf{R}^{3}, \zeta \in \mathbf{R}^{2}$ are, respectively, vectors representing phase variables and control parameters. Assume that $f$ is of class $C^{\infty}$ in $\mathbf{R}^{3} \times \mathbf{R}^{2}$. Suppose that (8) has an equilibrium point $x=x_{0}$ at $\zeta=\zeta_{0}$ and, denoting the variable $x-x_{0}$ also by $x$, write

$$
F(x)=f\left(x, \zeta_{0}\right)
$$

as

$$
\begin{aligned}
F(x)= & A x+\frac{1}{2} B(x, x)+\frac{1}{6} C(x, x, x)+\frac{1}{24} D(x, x, x, x) \\
& +\frac{1}{120} E(x, x, x, x, x)+O\left(\|x\|^{6}\right),
\end{aligned}
$$


where $A=f_{x}\left(0, \zeta_{0}\right)$, and, for $i=1,2,3$,

$$
\begin{aligned}
B_{i}(x, y) & =\left.\sum_{j, k=1}^{3} \frac{\partial^{2} F_{i}(\xi)}{\partial \xi_{j} \partial \xi_{k}}\right|_{\xi=0} x_{j} y_{k}, \\
C_{i}(x, y, z) & =\left.\sum_{j, k, l=1}^{3} \frac{\partial^{3} F_{i}(\xi)}{\partial \xi_{j} \partial \xi_{k} \partial \xi_{l}}\right|_{\xi=0} x_{j} y_{k} z_{l} .
\end{aligned}
$$

Suppose that $\left(x_{0}, \zeta_{0}\right)$ is an equilibrium point of (8), where the Jacobian matrix $\mathbf{A}$ has a pair of purely imaginary eigenvalues $\lambda_{2,3}= \pm i \omega_{0},\left(\omega_{0}>0\right)$ and admits no other eigenvalue with zero real part. Let $\mathbf{T}^{c}$ be the generalized eigenspace of $\mathbf{A}$ corresponding to $\lambda_{2,3}$. By this it means the largest subspace invariant by $\mathbf{A}$ on which the eigenvalues are $\lambda_{2,3}$.

Let $p, q \in \mathbf{C}^{3}$ be vectors such that

$$
\mathbf{A} q=i \omega_{0} q, \quad \mathbf{A}^{\mathrm{T}} p=-i \omega_{0} p, \quad\langle p, q\rangle=\sum_{i=1}^{3} \bar{p}_{i} q_{i}=1,
$$

where $\mathbf{A}^{\mathrm{T}}$ is the transpose of the matrix $\mathbf{A}$. Any vector $y \in \mathbf{T}^{c}$ can be represented as $y=\omega q+\bar{\omega} \bar{q}$, where $\omega=\langle p, y\rangle \in \mathbf{C}$. The two-dimensional center manifold associated with the eigenvalues $\lambda_{2,3}= \pm i \omega_{0}$ can be parameterized by the variables $\omega$ and $\bar{\omega}$ by means of an immersion of the form $x=H(\omega, \bar{\omega})$, where $H: \mathbf{C}^{2} \rightarrow \mathbf{R}^{3}$ has a Taylor expansion of the form

$$
H(\omega, \bar{\omega})=\omega q+\bar{\omega} \bar{q}+\sum_{2 \leq j+k \leq 5} \frac{1}{j ! k !} h_{j k} \omega^{j} \bar{\omega}^{k}+O\left(|\omega|^{6}\right),
$$

with $h_{j k} \in \mathbf{C}^{3}$ and $h_{j k}=\bar{h}_{k j}$. Substituting this expression into (8) we obtain the following differential equation:

$$
H_{\omega} \omega^{\prime}+H_{\bar{\omega}} \bar{\omega}^{\prime}=F(H(\omega, \bar{\omega})),
$$

where $F$ is given by (9). The complex vectors $h_{j k}$ are obtained solving the system of linear equations defined by the coefficients of (14), taking into account the coefficients of $F$, so that system (14), on the chart $\omega$ for a central manifold, writes as follows:

$$
\omega^{\prime}=i \omega_{0} \omega+\frac{1}{2} G_{21} \omega|\omega|^{2}+\frac{1}{12} G_{32} \omega|\omega|^{4}+O\left(|\omega|^{6}\right),
$$

with $G_{j k} \in \mathbf{C}$.

The first Lyapunov coefficient $l_{1}$ is defined by

$$
l_{1}=\frac{1}{2} \operatorname{Re} G_{21},
$$

where $G_{21}=\left\langle p, H_{21}\right\rangle, H_{21}=C(q, q, \bar{q})+B\left(\bar{q}, h_{20}\right)+2 B\left(q, h_{11}\right)$, $h_{20}=\left(2 i \omega_{0} \mathbf{I}_{3}-\mathbf{A}\right)^{-1} B(q, q), h_{11}=-\mathbf{A}^{-1} B(q, \bar{q})$, and $\mathbf{I}_{3}$ is the unit $3 \times 3$ matrix.

The second Lyapunov coefficient is defined by

$$
l_{2}=\frac{1}{12} \operatorname{Re} G_{32},
$$

where $G_{32}=\left\langle p, H_{32}\right\rangle, H_{32}=6 B\left(h_{11}, h_{21}\right)+B\left(\bar{h}_{20}, h_{30}\right)+$ $3 B\left(\bar{h}_{21}, \bar{h}_{20}\right)+3 B\left(q, h_{22}\right)+2 B\left(\bar{q}, h_{31}\right)+6 C\left(q, h_{11}, h_{11}\right)+$ $3 C\left(q, \bar{h}_{20}, h_{20}\right)+3 C\left(q, q, \bar{h}_{21}\right)+6 C\left(q, \bar{q}, h_{21}\right)+6 C\left(\bar{q}, h_{20}, h_{11}\right)+$ $C\left(\bar{q}, \bar{q}, h_{30}\right)+D\left(q, q, q, \bar{h}_{20}\right)+6 D\left(q, q, \bar{q}, h_{11}\right)+3 D\left(q, \bar{q}, \bar{q}, h_{20}\right)+$ $E(q, q, q, \bar{q}, \bar{q})-6 G_{21} h_{21}-3 \bar{G}_{21} h_{21}$.

The complex vector $h_{21}$ can be found by solving the nonsingular $n+1$-dimensional system

$$
\left(\begin{array}{cc}
i \omega_{0} \mathbf{I}_{3}-\mathbf{A} & q \\
\bar{p} & 0
\end{array}\right)\left(\begin{array}{c}
h_{21} \\
s
\end{array}\right)=\left(\begin{array}{c}
H_{21}-G_{21} q \\
0
\end{array}\right),
$$

with the condition $\left\langle p, h_{21}\right\rangle=0$. Consider the following:

$$
\begin{aligned}
& h_{30}=(\left.3 i \omega_{0} \mathbf{I}_{3}-\mathbf{A}\right)^{-1}\left[3 B\left(q, h_{20}\right)+C(q, q, q)\right], \\
& h_{31}=\left(2 i \omega_{0} \mathbf{I}_{3}-\mathbf{A}\right)^{-1} \\
& \times\left[3 B\left(q, h_{21}\right)+B\left(\bar{q}, h_{30}\right)+3 B\left(h_{20}, h_{11}\right)\right. \\
&+3 C\left(q, q, h_{11}\right)+3 C\left(q, \bar{q}, h_{20}\right) \\
&\left.+D(q, q, q, \bar{q})-3 G_{21} h_{20}\right], \\
& h_{22}=-\mathbf{A}^{-1}\left[D(q, q, q, \bar{q})+4 C\left(q, \bar{q}, h_{11}\right)\right. \\
&+C\left(\bar{q}, \bar{q}, h_{20}\right)+C\left(q, q, \bar{h}_{20}\right) \\
&+2 B\left(h_{11}, h_{11}\right)+2 B\left(q, \bar{h}_{21}\right) \\
&\left.+2 B\left(\bar{q}, h_{21}\right)+B\left(\bar{h}_{20}, h_{20}\right)\right] .
\end{aligned}
$$

A Hopf point $\left(x_{0}, \zeta_{0}\right)$ of system (8) is an equilibrium point where the Jacobian matrix $\mathbf{A}$ has a pair of purely imaginary eigenvalues $\lambda_{2,3}= \pm i \omega_{0},\left(\omega_{0}>0\right)$, and the other eigenvalue $\lambda_{1} \neq 0$. From the Center Manifold Theorem, at a Hopf point, a two-dimensional center manifold is well defined; it is invariant under the flow generated by (8) and can be continued with arbitrary high class of differentiability to nearby parameter values.

A Hopf point is called transversal if the parameterdependent complex eigenvalues cross the imaginary axis with nonzero derivative. In a neighborhood of a transversal Hopf point with $l_{1} \neq 0$ the dynamic behavior of the system (8), reduced to the family of parameter-dependent continuations of the center manifold, is orbitally topologically equivalent to the following complex normal form: $\omega^{\prime}=(\eta+i \omega) \omega+l_{1} \omega|\omega|^{2}$, where $\omega \in \mathbf{C} . \eta, \omega$ and $l_{1}$ are real functions having derivatives of arbitrary higher order, which are continuations of $0, \omega_{0}$, and the first Lyapunov coefficient at the Hopf point. When $l_{1}<0\left(l_{1}>0\right)$ one family of stable (unstable) periodic orbits can be found on this family of manifolds, shrinking to an equilibrium point at the Hopf point.

A Hopf point of codimension 2 is a Hopf point, where $l_{1}$ vanishes. It is called transversal if $l_{1}=0$ and $\eta=0$ have transversal intersections, where $\eta=\eta(\mu)$ is the real part of the critical eigenvalues. In a neighborhood of a transversal Hopf point of codimension 2 with $l_{2} \neq 0$ the dynamic behavior of the system (8), reduced to the family of parameter-dependent continuations of the center manifold, is orbitally topologically 
equivalent to $\omega^{\prime}=\left(\eta+i \omega_{0}\right) \omega+\tau \omega|\omega|^{2}+l_{1} \omega|\omega|^{4}$, where $\eta$ and $\tau$ are unfolding parameters.

\section{Hopf Bifurcations at $E_{+}$}

In this section we will study the stability of $E_{+}$under the conditions $a=a_{0}$ and $b>0$. Then, using the notion of the previous section the multilinear symmetric functions corresponding to $f$ can be written as

$$
\begin{gathered}
B(x, y)=\left(x_{2} y_{3}+x_{3} y_{2}, 0,-x_{1} y_{2}-x_{2} y_{1}\right), \\
C(x, y, z)=(0,0,0) .
\end{gathered}
$$

The eigenvalues of $\mathbf{A}$ are

$$
\lambda_{1}=a-1, \quad \lambda_{2,3}= \pm i \omega_{0}= \pm i \sqrt{b} .
$$

And from (12) one has

$$
\begin{gathered}
q=\left(\frac{3 \sqrt{b}+(b-2) i}{b+4}, \frac{\sqrt{b}-2 i}{b+4}, 1\right), \\
p=\left(\frac{\sqrt{b}-2 i}{3-a}, \frac{-a \sqrt{b}+2 a i}{3-a}, \frac{2+\sqrt{b} i}{3-a}\right), \\
B(q, q)=\left(\frac{2 \sqrt{b}-4 i}{b+4}, 0, \frac{4(1-2 b)-2 \sqrt{b}(b-8) i}{(b+4)^{2}}\right), \\
B(q, \bar{q})=\left(\frac{2 \sqrt{b}}{b+4}, 0,-\frac{1}{b+4}\right), \\
h_{11}=-A^{-1} B(q, \bar{q})=\left(\frac{-1}{2 \sqrt{b}(b+4)}, \frac{-1}{2 \sqrt{b}(b+4)}, \frac{-2}{b+4}\right), \\
h_{20}=\left(2 i \omega_{0} I_{3}-A\right)^{-1} B(q, q)=\left(k_{1}+k_{2} i, k_{3}+k_{4} i, k_{5}+k_{6} i\right),
\end{gathered}
$$

where

$$
\begin{gathered}
k_{1}=\frac{2(1-2 a)\left(b^{2}+16 b-9\right)-3 b(b+52)}{\sqrt{b}(b+4)^{2}\left(4 a^{2}-4 a+9 b+1\right)}, \\
k_{2}=\frac{-(1-2 a)(b+52)-6\left(b^{2}+16 b-9\right)}{(b+4)^{2}\left(4 a^{2}-4 a+9 b+1\right)}, \\
k_{3}=\frac{3 b(b-8)-6 \sqrt{b}(b+4)-8(1-2 a)}{\sqrt{b}(b+4)^{2}\left(4 a^{2}-4 a+9 b+1\right)}, \\
k_{4}=\frac{24 \sqrt{b}+\sqrt{b}(1-2 a)(b-8)-2(b+4)(1-2 a)}{(b+4)^{2}\left(4 a^{2}-4 a+9 b+1\right)}, \\
k_{5}=\frac{4\left(4 a^{2} b+a b^{2}-15 a b-2 a^{2}+18 a+6 b-24\right)}{(b+4)^{2}\left(4 a^{2}-4 a+9 b+1\right)},
\end{gathered}
$$

$$
\begin{gathered}
k_{6}=\frac{2\left(8 a^{2} b-a b^{2}-8 a b-4 a^{2}+22 a+18 b-58 b-10\right)}{(b+4)^{2}\left(4 a^{2}-4 a+9 b+1\right)}, \\
B\left(q, h_{11}\right)=\left(m_{1}+m_{2} i, 0, m_{3}+m_{4} i\right), \\
B\left(\bar{q}, h_{20}\right)=\left(m_{5}+m_{6} i, 0, m_{7}+m_{8} i\right),
\end{gathered}
$$

where

$$
\begin{aligned}
& m_{1}=\frac{-5 b-4}{2 \sqrt{b}(b+4)^{2}}, \quad m_{2}=\frac{4}{(b+4)^{2}}, \\
& m_{3}=\frac{2}{(b+4)^{2}}, \quad m_{4}=\frac{b-4}{(b+4)^{2}}, \\
& m_{5}=\frac{(b+4) k_{3}+\sqrt{b} k_{5}+2 k_{6}}{b+4}, \\
& m_{6}=\frac{(b+4) k_{4}-2 k_{5}+\sqrt{b} k_{6}}{b+4}, \\
& m_{7}=\frac{2 k_{2}-\sqrt{b} k_{1}-3 \sqrt{b} k_{3}+(b-2) k_{4}}{b+4}, \\
& m_{8}=\frac{2 k_{1}+\sqrt{b} k_{2}+3 \sqrt{b} k_{4}+(b-2) k_{3}}{b+4} .
\end{aligned}
$$

We can get

$$
\begin{aligned}
H_{21}= & C(q, q, \bar{q})+B\left(\bar{q}, h_{20}\right)+2 B\left(q, h_{11}\right) \\
= & \left(n_{1}+n_{2} i, 0, n_{3}+n_{4} i\right), \\
G_{21}= & \left\langle p, H_{21}\right\rangle=\frac{\sqrt{b} n_{1}+2 n_{2}+2 n_{3}+\sqrt{b} n_{4}}{3-a} \\
& -\frac{2 n_{1}-\sqrt{b} n_{2}-\sqrt{b} n_{3}-2 n_{4}}{3-a} i,
\end{aligned}
$$

where

$$
\begin{array}{ll}
n_{1}=2 m_{1}+m_{5}, & n_{2}=2 m_{2}+m_{6}, \\
n_{3}=2 m_{3}+m_{7}, & n_{4}=2 m_{4}+m_{8} .
\end{array}
$$

Now it remains only to verify the transversality condition of the Hopf bifurcation. In order to do so, consider the family of differential equation (1) regarded as dependent on the parameter $a$. The real part $\xi$, of the pair of complex eigenvalues at the critical parameter $a=a_{0}$, verifies

$$
\xi^{\prime}\left(a_{0}\right)=\operatorname{Re}\left\langle p,\left.\frac{d \mathbf{A}}{d a}\right|_{a=a_{0}} q\right\rangle=\frac{b}{b+4}>0 .
$$

Since $\xi^{\prime}\left(a_{0}\right) \neq 0$, the transversality condition at the Hopf point holds.

Using these calculations we prove the next theorem.

Theorem 2. Consider the three-parameter family of differential equations (1). The first Lyapunov coefficient associated with the equilibrium $E_{+}$is given by

$$
l_{1}\left(a_{0}, b\right)=\frac{N_{1}}{72 \sqrt{b}(b+1)(b+4)^{4}},
$$




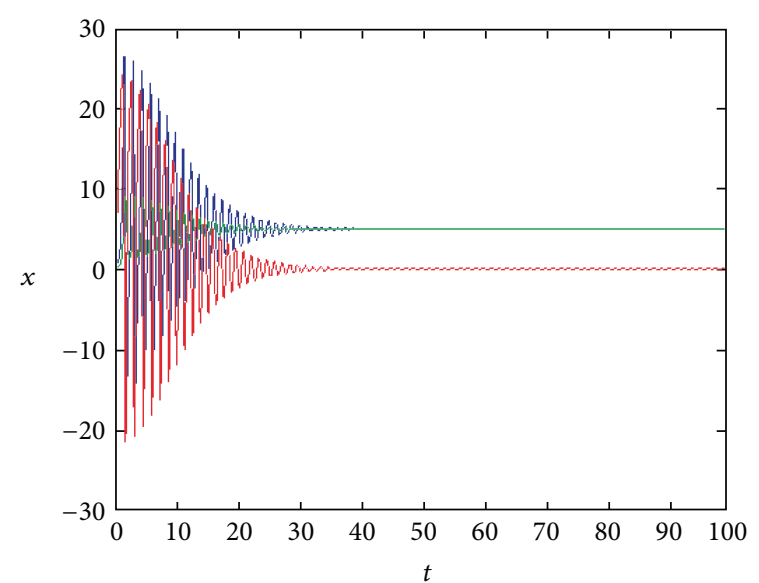

(a)

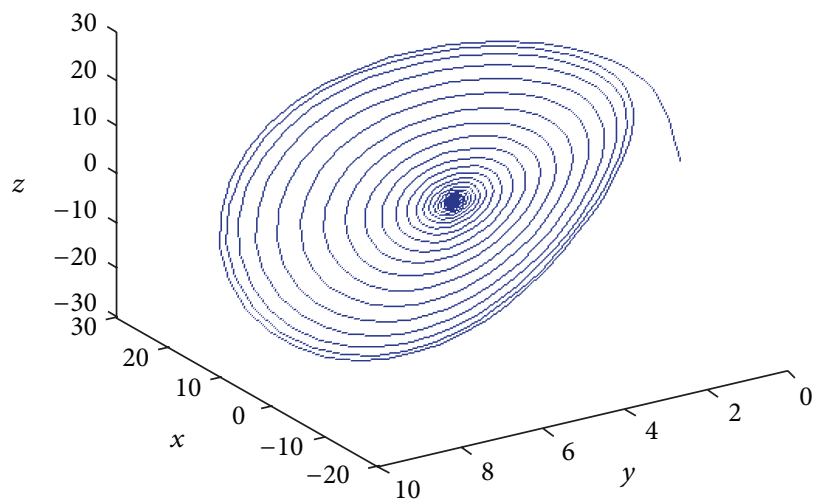

(b)

FIGURE 1: Time history and phase diagram of system (1) with $a=-1.4, b=25$.

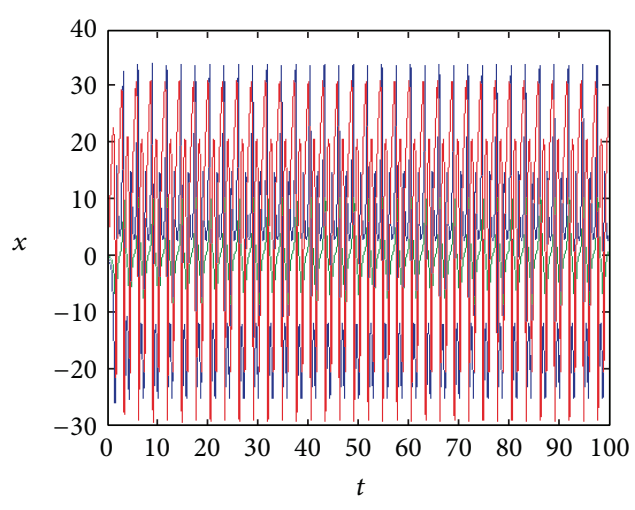

(a)

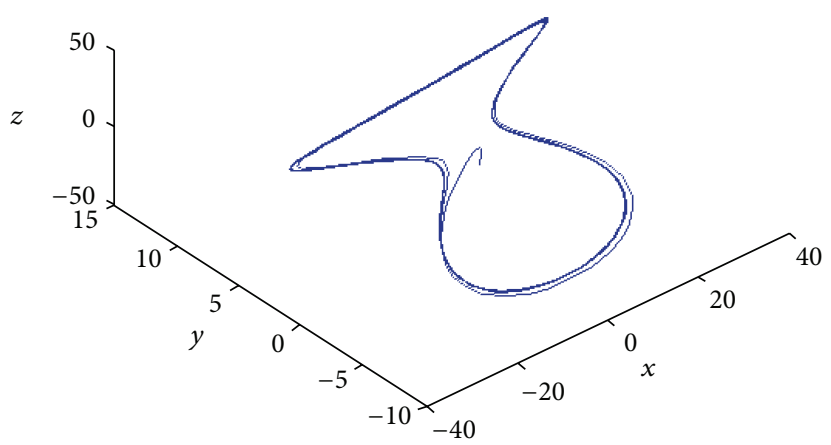

(b)

Figure 2: Time history and phase diagram of system (1) with $a=-1, b=25$.

where

$$
\begin{aligned}
N_{1}= & 3(\sqrt{b}+2) b^{4}+(47 \sqrt{b}+72) b^{3} \\
& +2(193 \sqrt{b}+122) b^{2}+(61-155 \sqrt{b}) b-38 \sqrt{b}+368
\end{aligned}
$$

As $b>0$ then $l_{1}\left(a_{0}, b\right)>0$, so the system (1) has a transversal Hopf point at $E_{+}$when $a=a_{0}$ and $b>0$. More precisely, Hopf point at $E_{+}$is unstable and for each $a>a_{0}$, but close to $a_{0}$, there exists an unstable periodic orbit near the asymptotically stable equilibrium point $E_{+}$.

Next, we will give a numerical example of system (1). Let $b=25$; we can calculate the Hopf bifurcation value $a_{0}=-1$. The equilibrium is stable when $a=-1.4<a_{0}$ and unstable when $a=-0.8>a_{0}$, as shown in Figures 1 and 3, respectively. From the formulas in previous section, we have $\xi^{\prime}\left(a_{0}\right)>0$, $l_{1}>0$. Thus, the periodic solutions bifurcating from the equilibrium point $E_{+}$are subcritical and unstable (Figure 2).

When the parameter $b=25$ is fixed while parameter $a$ is varied in the interval $[-1.5,-0.7]$, some different dynamical behaviors of system (1) are obtained. The bifurcation diagram and the Lyapunov exponent spectrum of system (1) in terms of the parameter $a$ are depicted in Figure 4. With the analysis performed here one can find the Hopf bifurcation at the equilibrium $E_{+}$does occur when $a=-1$.

\section{Hopf Bifurcation Control}

In this section, we will design control laws such that our feedback system undergoes a supercritical and stable Hopf bifurcation. To accomplish the control of Hopf bifurcation in the system (1), we design the controller which has the following structure:

$$
u=k(x-y)^{3}
$$

where $k$ is the control gain. In the following, we will study the stability of $E_{+}$in the controlled system:

$$
\begin{aligned}
& \dot{x}=y z+a(x-y), \\
& \dot{y}=x-y, \\
& \dot{z}=b-x y+u .
\end{aligned}
$$




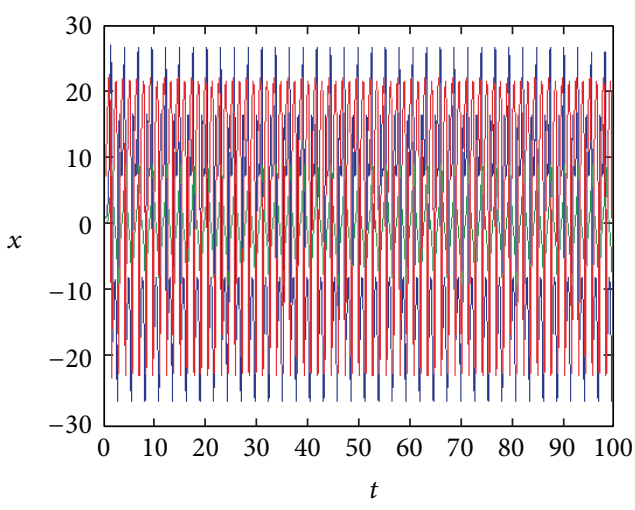

(a)

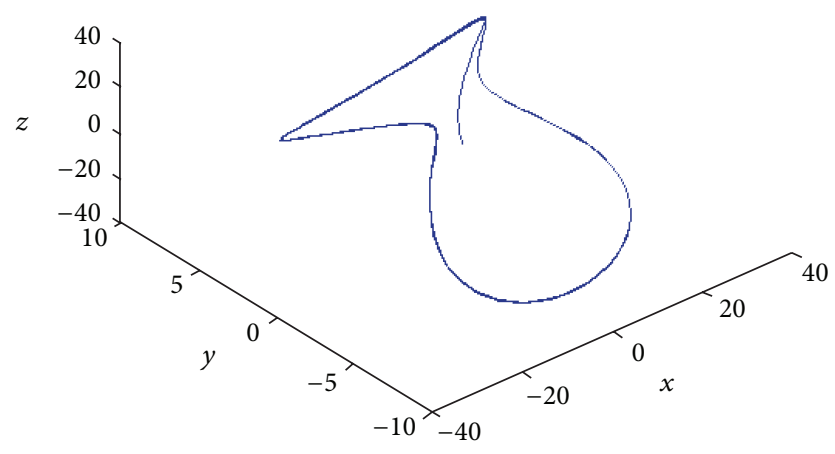

(b)

FIgURE 3: Time history and phase diagram of system (1) with $a=-0.8, b=25$.

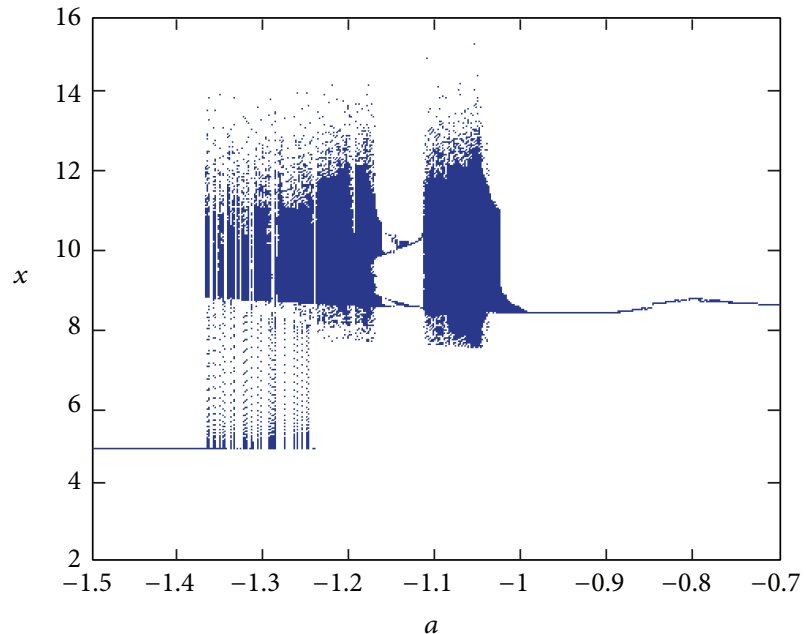

(a)

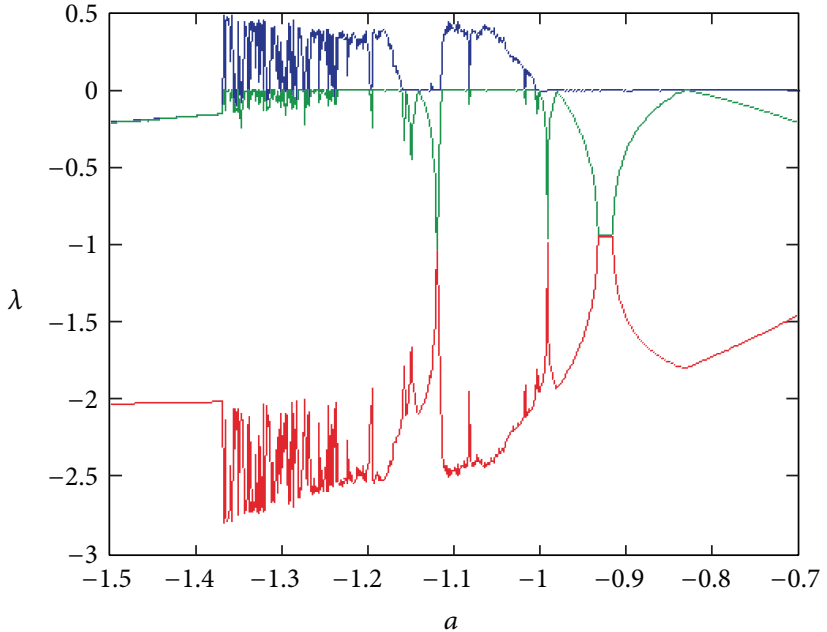

(b)

Figure 4: Nonlinear dynamics of system (1) for specific values $b=25$ versus the control parameter $a$. (a) Bifurcation diagram of $y$; (b) Lyapunov exponent spectrum.

Then, using the notion of the previous section the multilinear symmetric functions corresponding to $f$ can be written as

$$
\begin{aligned}
B(x, y)=\left(x_{2} y_{3}+x_{3} y_{2}, 0,-x_{1} y_{2}-x_{2} y_{1}\right), & \\
C(x, y, z)=(0,0,3 k & \left(2 x_{1} y_{1} z_{1}-2 x_{2} y_{2} z_{2}-x_{1} y_{1} z_{2}\right. \\
& -x_{1} y_{2} z_{1}-x_{2} y_{1} z_{1}+x_{1} y_{2} z_{2} \\
& \left.\left.+x_{2} y_{1} z_{2}+x_{2} y_{2} z_{1}\right)\right) .
\end{aligned}
$$

By direct calculation, we can get

$$
C(q, q, \bar{q})=\left(0,0, \frac{3 k b[3 \sqrt{b}+(b-2) i]}{(b+4)^{2}}\right)
$$

The first Lyapunov coefficient associated with the equilibrium $E_{+}$is given by

$$
l_{1}\left(a_{0}, b, k\right)=\frac{N_{2}(b+1)}{72(b+4)^{4}},
$$

where

$$
\begin{aligned}
N_{2}= & 27 k b^{11 / 2}+1620 k b^{7 / 2}+2382 b-45 b^{4}+1728 k b^{3 / 2} \\
& +3024 k b^{5 / 2}-1644 b^{3 / 2}-199 b^{5 / 2}+431 b^{2}-229 b^{3} \\
& +90 b^{7 / 2}+18 b^{9 / 2}+351 k b^{9 / 2}-1344 b^{1 / 2}+3176 .
\end{aligned}
$$

Obviously, the value of $l_{1}\left(a_{0}, b, k\right)$ is associated with $b$, $k$. We can adjust the positive and negative of the $l_{1}\left(a_{0}, b, k\right)$ through the change of the value of $k$. According to the last section, we know that the Hopf bifurcation at the equilibrium point $E_{+}$is subcritical and unstable. Let $b=25$ and obtain 


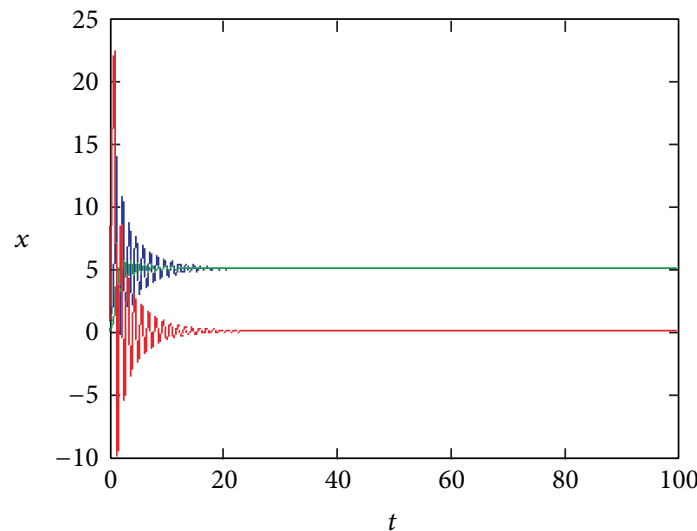

(a)

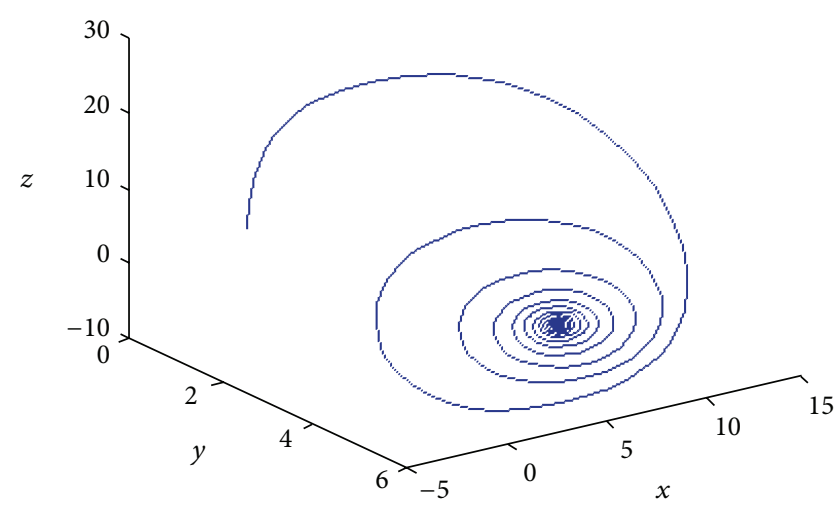

(b)

FIgURE 5: Time history and phase diagram of system (31) with $a=-1.5, b=25, k=-0.1$.

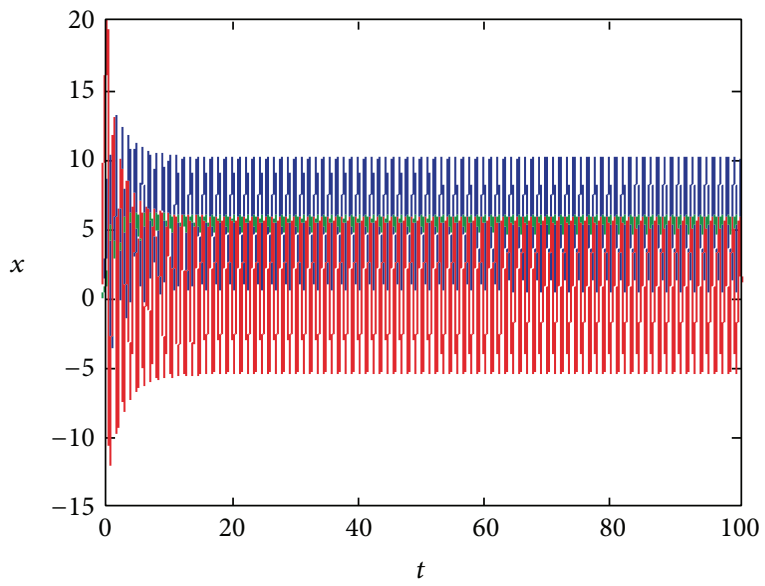

(a)

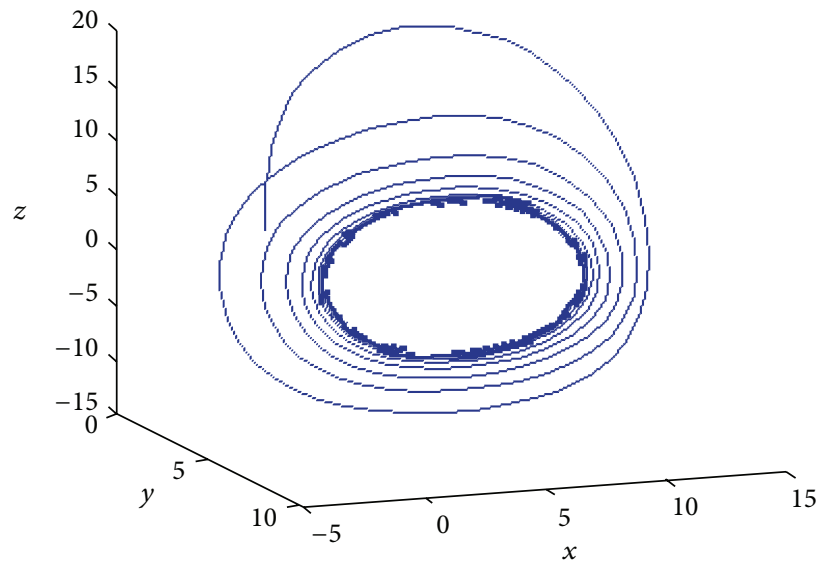

(b)

FIgURE 6: Time history and phase diagram of system (31) with $a=-0.5, b=25, k=-0.1$.

$l_{1}\left(a_{0}, b, k\right)=1.61637931 k+0.01550513$. If $k<-0.0096$, then $l_{1}\left(a_{0}, b, k\right)<0$. That is to say, if we get the value $k$ less than -0.0096 , we can control the unstable subcritical Hopf bifurcation to the stable supercritical Hopf bifurcation.

Next, we will give a numerical example of system (31). Let $b=25, k=-0.1$; we can calculate the first Lyapunov coefficient $l_{1}\left(a_{0}, b, k\right)=-0.1461<0$. Thus, the periodic solutions bifurcating from the equilibrium point $E_{+}$are supercritical and stable. The time history and phase diagram are shown in Figures 5 and 6, respectively.

Observe that the first Lyapunov coefficient vanishes if and only if $a=-1, b=25, k=-0.0096$. In the following theorem we study the sign of the second Lyapunov coefficient where the first coefficient vanishes.

Theorem 3. Consider the differential equations (31). The second Lyapunov coefficient associated with the equilibrium $E_{+}$ is given by
As $l_{2}<0$ and the transversality condition at the Hopf point holds then system (31) has a transversal Hopf point of codimension 2 at $E_{+}$. More specifically, the Hopf point at $E_{+}$is stable.

Proof. Following the notation introduced in Section 3 and by direct calculations one has

$$
\begin{gathered}
C(q, q, \bar{q})=(0,0,-0.0128-0.0197 i), \\
H_{21}=(0,-1.2009-3.3663 i,-1.259+2.7 i), \\
G_{21}=0.0321 i, \\
h_{30}=(0.0006+0.0003 i, 0,-0.0009+0.0014 i), \\
h_{21}=(-0.3686-1.3069 i, 0,1.2862-0.6341 i), \\
h_{31}=(-0.2626-0.5323 i, 0.0448-0.1325 i, \\
0.2198+0.2225 i),
\end{gathered}
$$




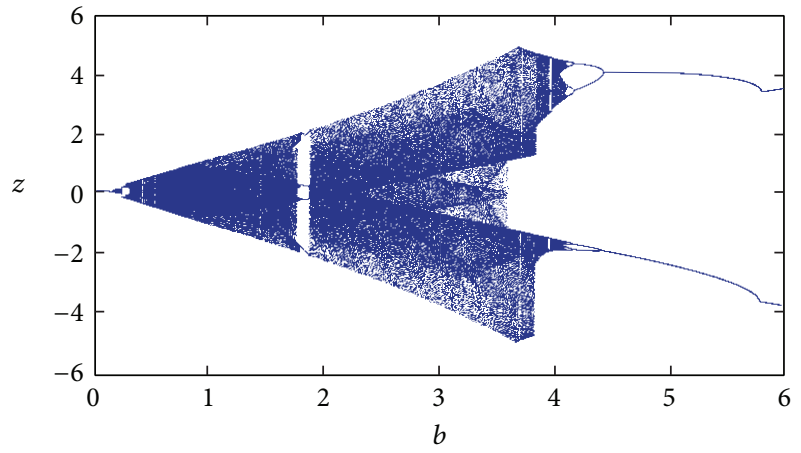

(a)

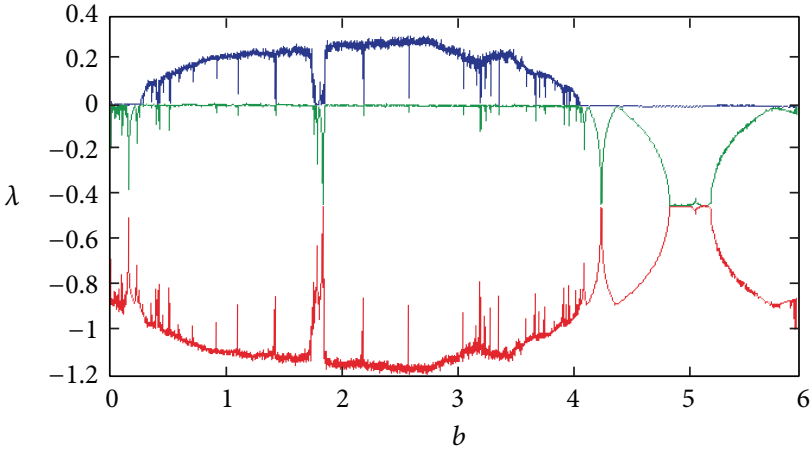

(b)

FIGURE 7: Nonlinear dynamics of system (1) for specific values $a=0.1$ versus the control parameter $b$. (a) Bifurcation diagram of $x$; (b) Lyapunov exponent spectrum.

$$
\begin{gathered}
h_{22}=(-0.0004-0.0885 i, 0.0444+0.044 i, \\
0.0436-0.133 i) \\
G_{32}=-0.2264+0.3751 i \\
l_{2}=\frac{1}{12} \operatorname{Re} G_{32}=-0.018869167 .
\end{gathered}
$$

\section{The Global Bifurcation Analysis}

For this system, bifurcation can easily be detected by examining graphs of $x$ versus each of the control parameters $a$ and $b$, respectively, if we fix the other one.

When the parameters $a=0.1$ are fixed, $b$ varies on the closed interval $[0,6]$. Figures $7(a)-7(b)$ show the bifurcation diagrams of the state $x$ and the corresponding Lyapunov exponent spectrum versus increasing $b$, respectively. While $b$ increases the system is undergoing some representative dynamical routes, such as period-doubling bifurcations, chaos, stable fixed points, and stable periodic loops.

As $b$ increases, system (1) is undergoing the flowing dynamical routes:

(1) if $0<b \leq 0.2, \lambda_{1}<0, \lambda_{2}<0, \lambda_{3}<0$, the system is stable;

(2) if $0.2 \leq b<1.7, \lambda_{1}>0, \lambda_{2}=0, \lambda_{3}<0$, the system is chaotic. But there are several periodic windows in the chaotic band;

(3) if $1.7 \leq b<1.9, \lambda_{1}=0, \lambda_{2} \leq 0, \lambda_{3}<0$, there is a reverse period-doubling bifurcation route with a flip bifurcation;

(4) if $1.9 \leq b<4.2, \lambda_{1}>0, \lambda_{2}=0, \lambda_{3}<0$, the system is chaotic. But there are several periodic windows in the chaotic band;

(5) if $4.2 \leq b \leq 6, \lambda_{1}=0, \lambda_{2} \leq 0, \lambda_{3}<0$, there is a very long reverse period-doubling bifurcation window.
When the parameters $b=2$ are fixed, $a$ varies on the closed interval $[0,0.6]$. Figures $8(a)-8(b)$ show the bifurcation diagrams of the state $x$ and the corresponding Lyapunov exponent spectrum versus increasing $a$, respectively. While $a$ increases the system is undergoing some representative dynamical routes, such as period-doubling bifurcations, chaos, and stable periodic loops.

As $a$ increases, system (1) is undergoing the flowing dynamical routes:

(1) if $0<a \leq 0.06, \lambda_{1}>0, \lambda_{2}=0, \lambda_{3}<0$, the system is chaotic;

(2) if $0.06 \leq a<0.08, \lambda_{1}=0, \lambda_{2} \leq 0, \lambda_{3}<0$, there is a reverse period-doubling bifurcation route with a flip bifurcation;

(3) if $0.08 \leq a<0.38, \lambda_{1}>0, \lambda_{2}=0, \lambda_{3}<0$, the system is chaotic. But there are several periodic windows in the chaotic band;

(4) if $0.38 \leq b<0.6, \lambda_{1}=0, \lambda_{2} \leq 0, \lambda_{3}<0$, there is a very long reverse period-doubling bifurcation window.

Let $\Delta_{1}=0, \Delta_{2}=0, \Delta_{3}=0$; we use MATLAB to draw the stability region on the parameter plane $a-b$, as shown in Figure 9. In the figure, the symbol $L_{i}, i=1,2,3$ represents $\Delta_{i}=0, i=1,2,3$. If $\Delta_{1}>0, \Delta_{2}>0$ and $\Delta_{3}>0$, then the equilibrium $E_{+}$is asymptotically stable. If $\Delta_{1}>0, \Delta_{3}>0$ and $\Delta_{2}=0$, then system (1) has a transversal Hopf point at $E_{+}$. And in regions (I) and (IV): $\Delta_{1}>0, \Delta_{2}<0, \Delta_{3}<0$, in regions (II) and (III): $\Delta_{1}>0, \Delta_{2}>0, \Delta_{3}>0$, in region (V): $\Delta_{1}<0, \Delta_{2}>0, \Delta_{3}>0$, and in region (VI): $\Delta_{1}<0, \Delta_{2}<0$, $\Delta_{3}<0$. All of the points are stable in regions (II) and (III) and in the other regions are unstable (Figure 10).

\section{Sliding Mode Control of Chaotic Vibrations}

7.1. The Design of the Controller. We designed a sliding surface with good nature and made the system possess the desired properties when it limitations on the sliding surface. Then to facilitate control, make the system reach the sliding surface 


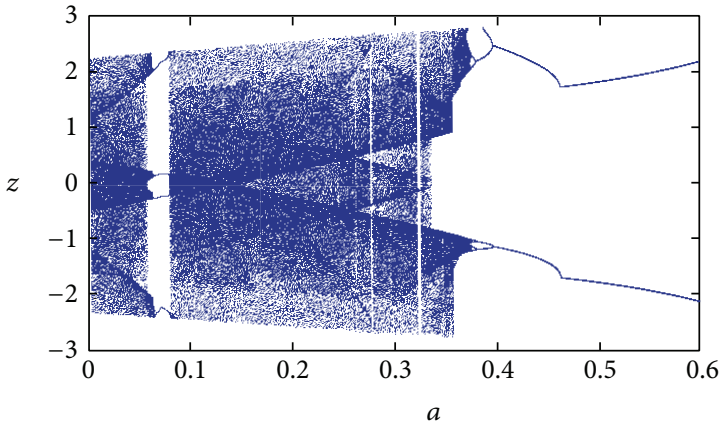

(a)

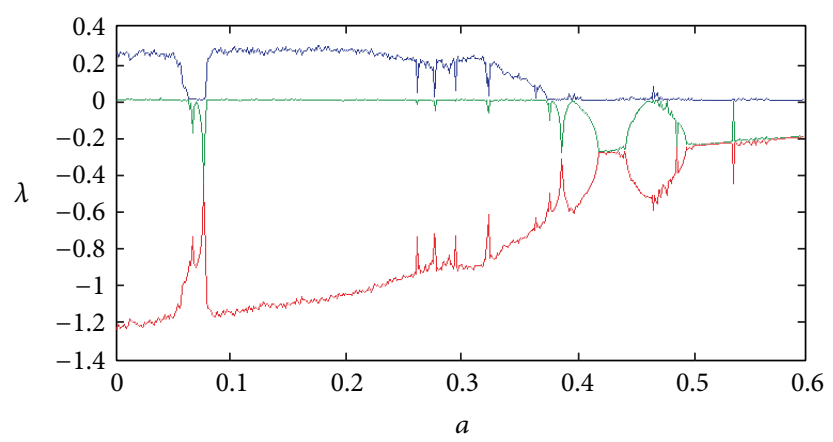

(b)

FIGURE 8: Nonlinear dynamics of system (1) for specific values $a=2$ versus the control parameter $a$. (a) Bifurcation diagram of $x$; (b) Lyapunov exponent spectrum.

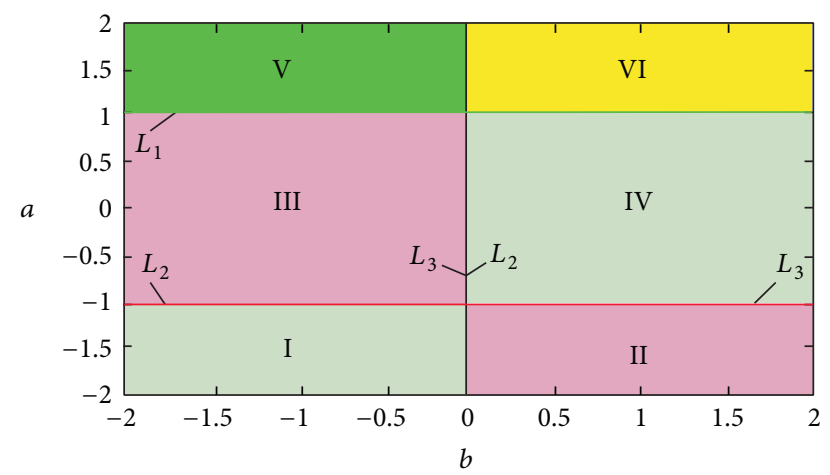

FIGURE 9: The stable region on the parameter plane $(a, b)$.

and keep sliding. After joining the controller, the system (1) has the following form:

$$
\begin{aligned}
& \dot{x}=y z+0.1(x-y)+d_{1}+u_{1}, \\
& \dot{y}=x-y+d_{2}+u_{2}, \\
& \dot{z}=2-x y+d_{3}+u_{3},
\end{aligned}
$$

where $u_{1}, u_{2}$, and $u_{3}$ are control inputs. If we join the reasonable controller, we can control the chaos system to the required range or the fixed point.

Define the following matrix:

$$
\begin{array}{cc}
\mathbf{A}=\left(\begin{array}{ccc}
0.1 & -0.1 & 0 \\
1 & -1 & 0 \\
0 & 0 & 0
\end{array}\right), \quad \mathbf{B}=\left(\begin{array}{lll}
1 & 0 & 0 \\
0 & 1 & 0 \\
0 & 0 & 1
\end{array}\right), \\
\mathbf{d}=\left(\begin{array}{l}
d_{1} \\
d_{2} \\
d_{3}
\end{array}\right), \quad \mathbf{g}=\left(\begin{array}{c}
y z \\
0 \\
-x y
\end{array}\right),
\end{array}
$$

where $\mathbf{A}$ is the linear matrix of the system, $\mathbf{B}$ is the control matrix, $\mathbf{d}$ is the bounded perturbation matrix, and $\mathbf{g}$ is the nonlinear matrix of the system. The purpose of control is to let the system state $\mathbf{x}=\left[x_{1}, x_{2}, x_{3}\right]^{\mathrm{T}}$ to track a time-varying state $\mathbf{x}_{d}=\left[x_{d 1}, x_{d 2}, x_{d 3}\right]^{\mathrm{T}}$. So, we can define the tracking error

$$
\mathbf{e}=\mathbf{x}-\mathbf{x}_{d}
$$

The error dynamic system can be written as

$$
\dot{\mathbf{e}}=\dot{\mathbf{x}}-\dot{\mathbf{x}}_{d}=\mathbf{A x}+\mathbf{B g}+\mathbf{B u}+\mathbf{d}-\dot{\mathbf{x}}_{d} .
$$

Define the time-varying proportional integral sliding mode surface

$$
\mathbf{S}=\mathbf{K e}-\int_{0}^{t} \mathbf{K}(\mathbf{A}-\mathbf{B L}) \mathbf{e}(\tau) d \tau
$$

where $\mathbf{K} \in \mathbf{R}^{3 \times 3}, \operatorname{det}(\mathbf{K B}) \neq 0$. For the convenience of calculation, we get $\mathbf{K}=\operatorname{diag}(1,1,1)$. The additional matrix $\mathbf{L} \in \mathbf{R}^{3 \times 3}$, and $\mathbf{A}-\mathbf{B L}$ is negative definite matrix. The equation $\mathbf{S}=\dot{\mathbf{S}}=0$ must be satisfied under the sliding mode, where

$$
\dot{\mathbf{S}}=\mathbf{K B g}+\mathbf{K B L e}+\mathbf{K B u}+\mathbf{K d}+\mathbf{K A x} \mathbf{x}_{d}-\mathbf{K}_{d}
$$

In order to satisfy the sliding conditions, the following controller is designed:

$$
\begin{aligned}
\mathbf{u}= & -[\mathbf{g}+\mathbf{L e}]-(\mathbf{K B})^{-1}\left[\mathbf{K A x} \mathbf{x}_{d}-\mathbf{K} \dot{\mathbf{x}}_{d}\right] \\
& -(\mathbf{K B})^{-1}[\varepsilon+\|\mathbf{K B g}\|] \operatorname{sign}(\mathbf{S}),
\end{aligned}
$$

where $\operatorname{sign}(\mathbf{S})$ is symbolic function.

Proposition 4. The controller (44) can make the system (38) reach the sliding mode $\mathbf{S}=0$ in a limited time if the constant $\varepsilon$ satisfied the inequality $\varepsilon>\delta_{1}+\delta_{2}+1$, where $\delta_{1}, \delta_{2}$ are arbitrary small positive numbers. The state variables and the selected reference state $\mathbf{x}_{d}$ are identical. 


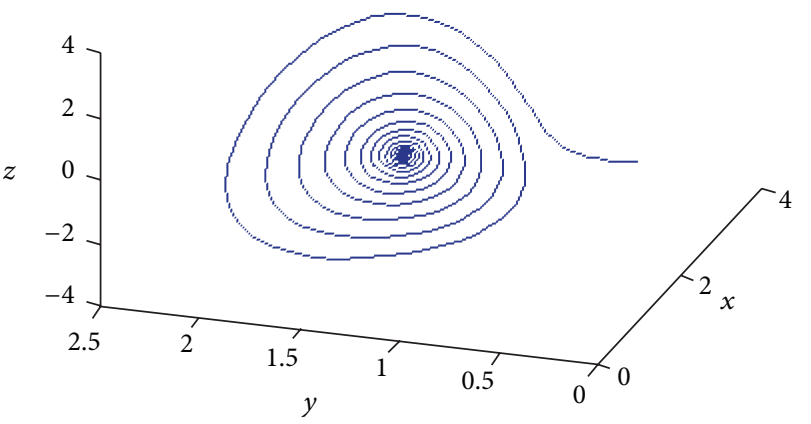

(a) $a=-1.5, b=1.5$

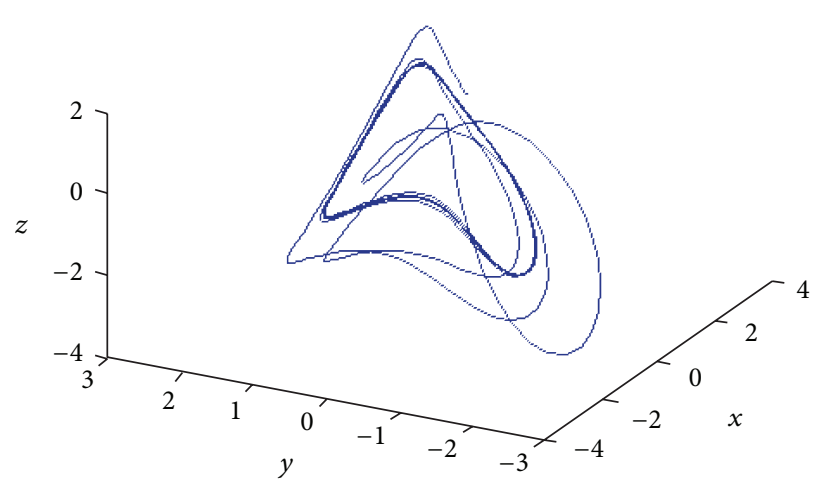

(c) $a=0.5, b=1.5$

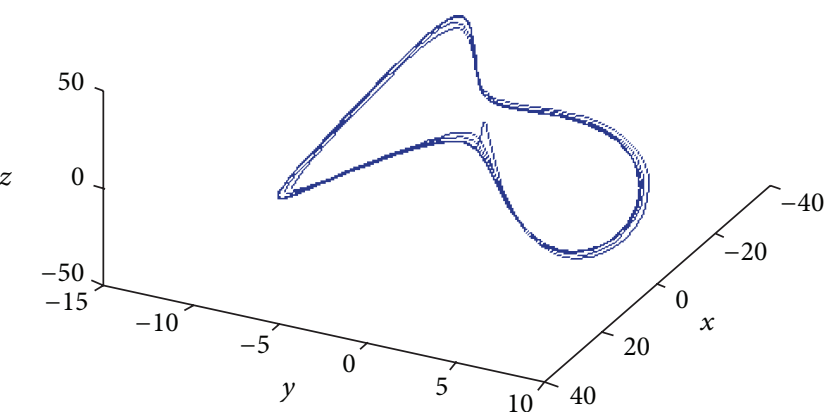

(b) $a=-1, b=2$

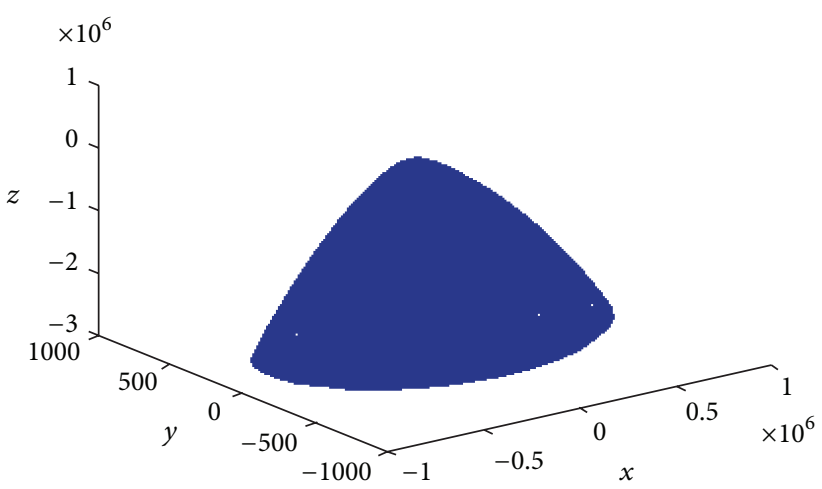

(d) $a=1.5, b=-1.5$

Figure 10: Phase diagram of system (1).

Proof. Construct the Lyapunov function $V=\mathbf{S}^{\mathrm{T}} \mathbf{S}=\sum_{i=1}^{3} \mathbf{S}_{i}^{2}$; according to (42), (43), and (44) one has

$$
\begin{aligned}
\mathbf{S}^{\mathrm{T}} \dot{\mathbf{S}} & =\mathbf{S}^{\mathrm{T}}\left(\mathbf{K B g}+\mathbf{K B L e}+\mathbf{K B u}+\mathbf{K d}+\mathbf{K A \mathbf { x }} \mathbf{x}_{d}-\mathbf{K} \dot{\mathbf{x}}_{d}\right) \\
& =\mathbf{S}^{\mathrm{T}}[\mathbf{K d}-(\varepsilon+\|\mathbf{K B g}\|) \operatorname{sign}(\mathbf{S})] \leq \mathbf{S}^{\mathrm{T}}[\mathbf{d}-\varepsilon \operatorname{sign}(\mathbf{S})] \\
& \leq \sum_{i=1}^{3}\left|\mathbf{S}_{i}\right| \delta_{1}+\sum_{i=1}^{3}\left|\mathbf{S}_{i}\right| \delta_{2}-\sum_{i=1}^{3}\left|\mathbf{S}_{i}\right| \varepsilon=\left|\delta_{1}+\delta_{2}-\varepsilon\right| \sum_{i=1}^{3}\left|\mathbf{S}_{i}\right| \\
& <-\sum_{i=1}^{3}\left|\mathbf{S}_{i}\right| .
\end{aligned}
$$

By the same token, we get

$$
\dot{\mathbf{S}}^{\mathrm{T}} \mathbf{S}<-\sum_{i=1}^{3}\left|\mathbf{S}_{i}\right|, \quad \dot{V}=\dot{\mathbf{S}}^{\mathrm{T}} \mathbf{S}+\mathbf{S}^{\mathrm{T}} \dot{\mathbf{S}}<-2 \sum_{i=1}^{3}\left|\mathbf{S}_{i}\right| .
$$

So the proposition follows.

7.2. The Numerical Simulation. In the case of $u_{1}=u_{2}=u_{3}=$ 0 , the time domain charts of the state variables of system (38) are shown in Figure 11. Figure 11 illustrates that the system (38) has aperiodic motion state before control.

In order to control the system (38) to the target state, we select the eigenvalues of $\mathbf{A}-\mathbf{B L}$ which are $\mathbf{P}=[-5,-5,-5]$.
The pole-placement method is adopted to get the following matrix:

$$
\mathbf{L}=\left(\begin{array}{ccc}
5.1 & -0.1 & 0 \\
1 & 4 & 0 \\
0 & 0 & 5
\end{array}\right)
$$
lows:

Select proportional integral sliding mode surface as fol-

$$
\begin{aligned}
& S_{1}=e_{1}+\int_{0}^{t} 5 e_{1}(\tau) d \tau, \\
& S_{2}=e_{2}+\int_{0}^{t} 5 e_{2}(\tau) d \tau, \\
& S_{3}=e_{3}+\int_{0}^{t} 5 e_{3}(\tau) d \tau .
\end{aligned}
$$

Set the initial value $\left[x_{1}(0), x_{2}(0), x_{3}(0)\right]=[0.1,0.1,0.1]$ and the reference state $x_{d 1}=x_{d 2}=x_{d 3}=x_{d}$. The control signal is as follows:

$$
\begin{aligned}
& u_{1}=-y z-5.1 e_{1}+0.1 e_{2}+\dot{x}_{d}-(\varepsilon+|y z|) \operatorname{sign}\left(S_{1}\right), \\
& u_{2}=-e_{1}-4 e_{2}+\dot{x}_{d}-\varepsilon \operatorname{sign}\left(S_{2}\right), \\
& u_{3}=x y-5 e_{3}+\dot{x}_{d}-(\varepsilon+|x y|) \operatorname{sign}\left(S_{3}\right) .
\end{aligned}
$$

7.3. Control to the Fixed Point. We can stabilize the system (38) to any point by this method. In this paper, we select 


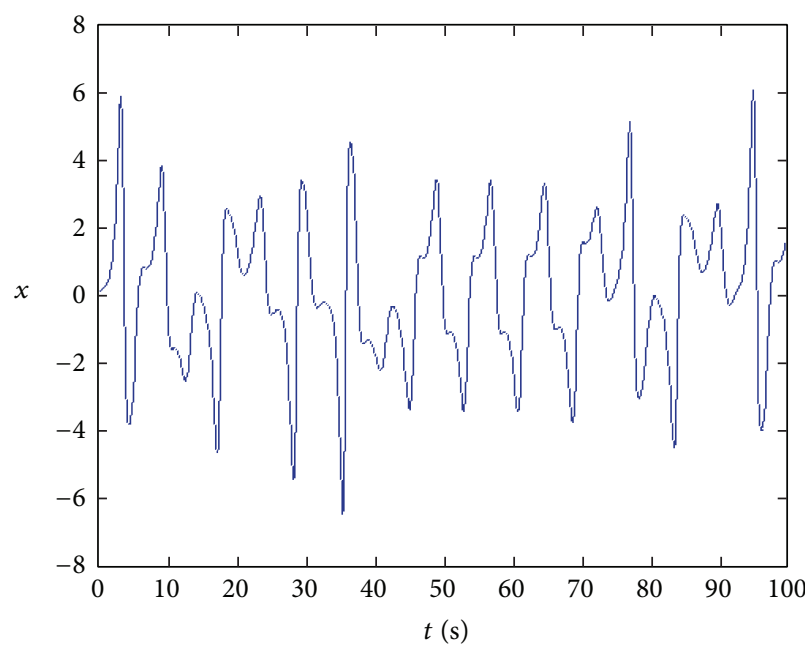

(a) Time domain chart of $x$ before control

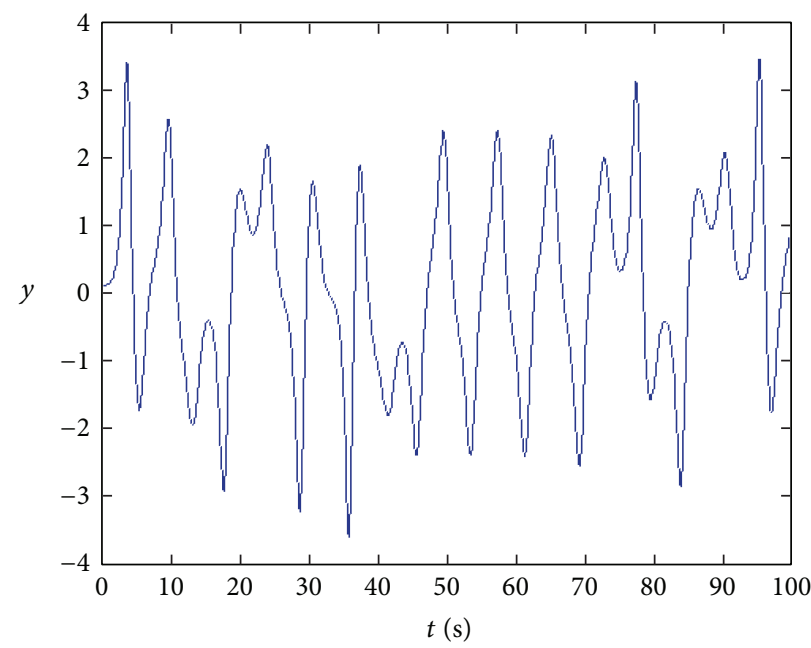

(b) Time domain chart of $y$ before control

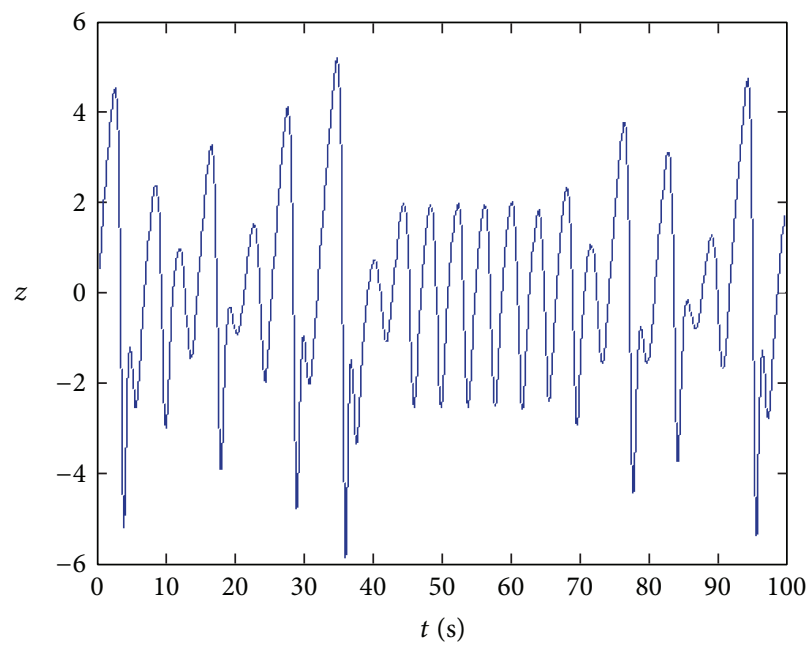

(c) Time domain chart of $z$ before control

FIGURE 11: Time domain charts of state variables before control.

the fixed point $[0,0,0]$, reference state $\mathbf{x}_{d}=0$, small parameter $\varepsilon=2$, and the initial value of the sliding mode surface $\left[S_{1}(0), S_{2}(0), S_{3}(0)\right]=[0.1,0.1,0.1]$. The controller $\mathbf{u}(t)$ is activated at $t=1 \mathrm{~s}$, the time domain charts of state variables and sliding surfaces as shown in Figures 12 and 13, respectively.

Figures 12 and 13 show that after joining the controller, the system (38) tracks reference state $[0,0,0]$ ultimately, and the sliding mode surface $\mathbf{S}$ becomes 0 . It is proved that the system (38) reached the sliding mode. The output curve of the controller will swing between upper and lower bounds in a certain base value; this suggests that the system error has reached zero. The reason of the fluctuations in the output of the controller is only that the small changes of $\mathbf{S}$ have caused the jump of the $\operatorname{sign}(\mathbf{S})$.

7.4. Control to the Periodic Orbit. We can stabilize the system (38) to the periodic orbit. In this paper, we select the reference state $\mathbf{x}_{d}=\sin (t)$. The controller $\mathbf{u}(t)$ is activated at $t=2 \mathrm{~s}$, the time domain charts of state variables as shown in Figure 14.

Obviously, the system (38) tracks reference state $\mathbf{x}_{d}=$ $\sin (t)$ to the periodic orbit ultimately.

\section{Concluding Remarks}

In this paper, a new autonomous system is studied in detail. Dynamical behaviors of the new system are analyzed, both theoretically and numerically, including some basic dynamical properties. By choosing an appropriate bifurcation parameter, we prove that Hopf bifurcation occurs when the bifurcation parameter passes through the critical value. The direction of the Hopf bifurcation and stability of the bifurcating periodic solutions are analyzed in detail. Besides, we designed a Hopf bifurcation controller so as to control the unstable subcritical Hopf bifurcation to the stable supercritical Hopf bifurcation. The stable region and 


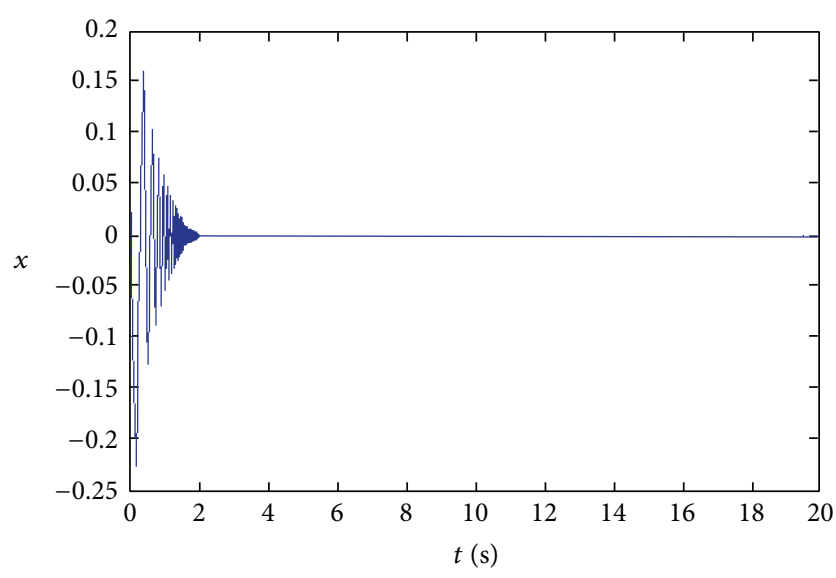

(a) Time domain chart of $x$ after control

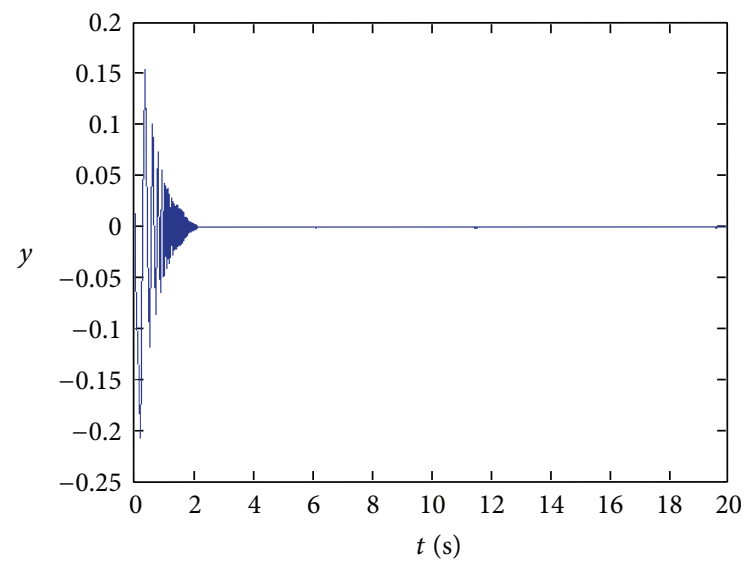

(b) Time domain chart of $y$ after control

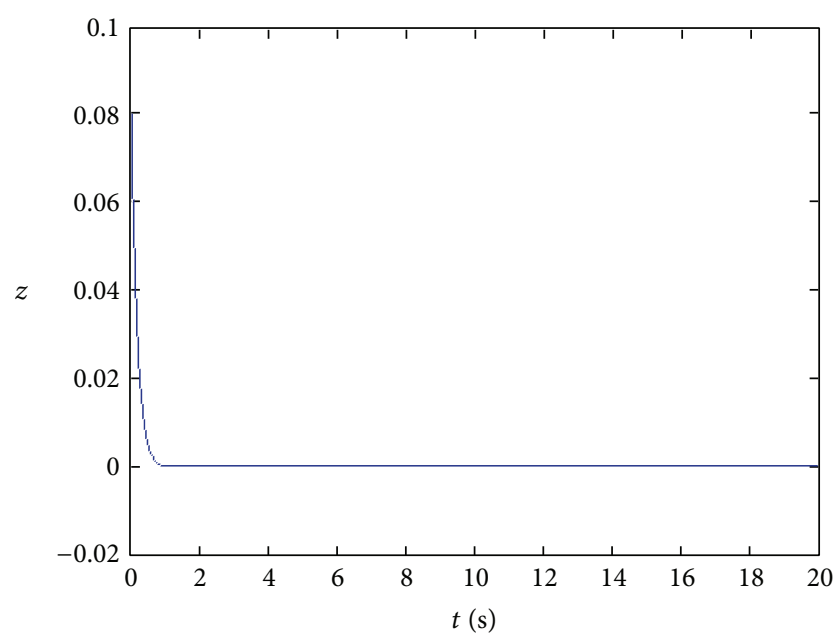

(c) Time domain chart of $z$ after control

FIGURE 12: Time domain charts of state variables after control.

the Hopf bifurcation boundaries are analyzed with the change of two parameters. At last, we designed a sliding surface with good nature and made the system possess the desired properties when it limitations on the sliding surface. And we eliminated the chaotic vibration by means of sliding mode method. Compared with other control methods, the

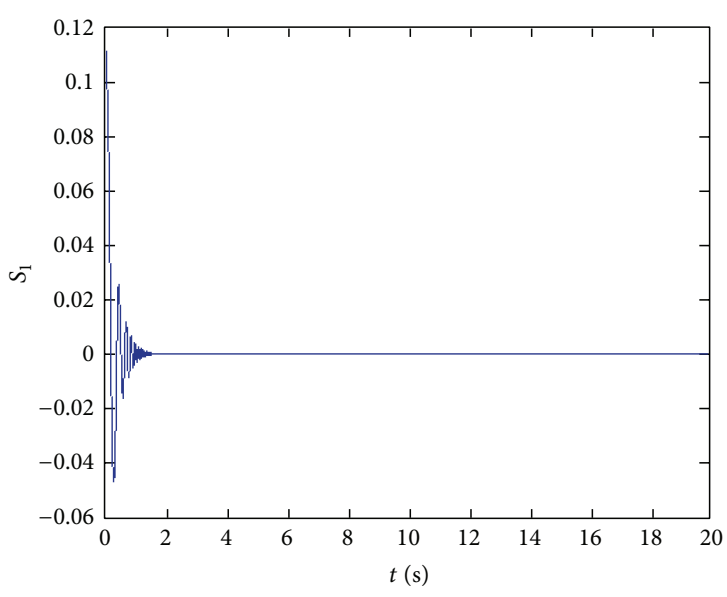

(a) Time domain chart of $S_{1}$ after control

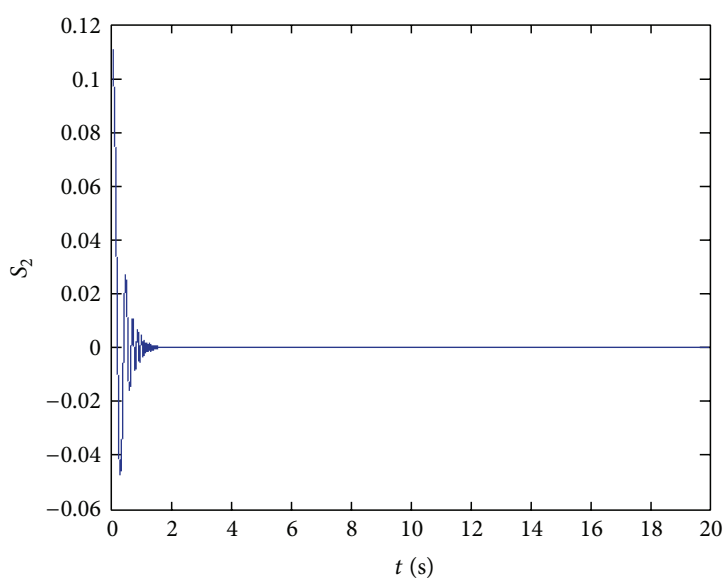

(b) Time domain chart of $S_{2}$ after control

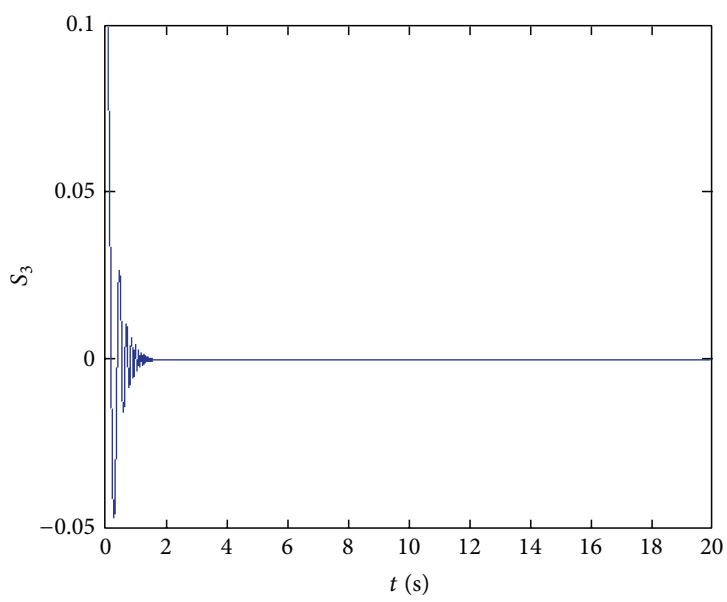

(c) Time domain chart of $S_{3}$ after control

FIGURE 13: Time domain charts of sliding surfaces after control.

sliding mode method can overcome the uncertainty of the system, has very strong robustness for the interference and unmodeled dynamics, and especially for nonlinear system control has better control effect. And the numerical simulations were presented to confirm the effectiveness of the controller. Apparently there are more interesting problems 


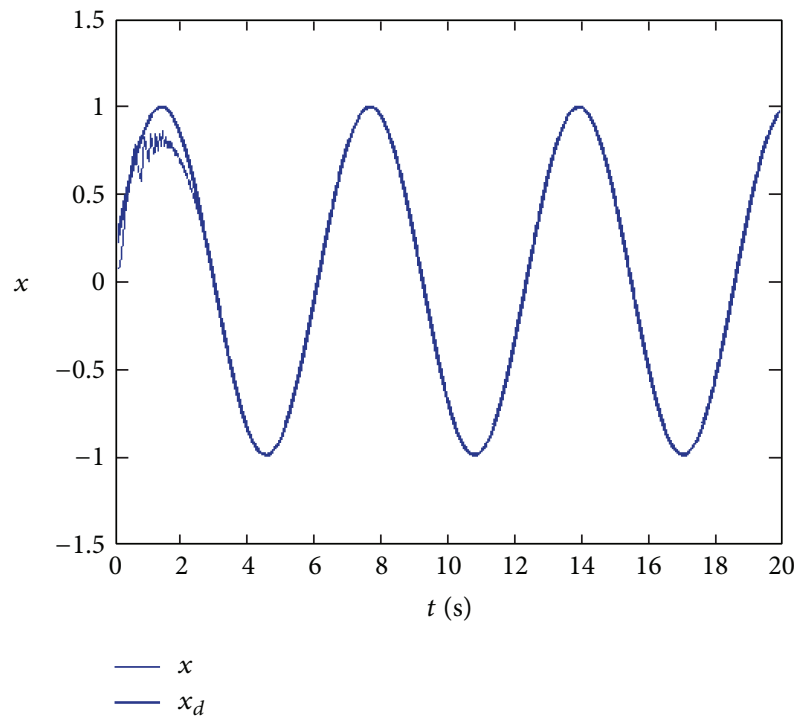

(a) Time domain chart of $x$ after control

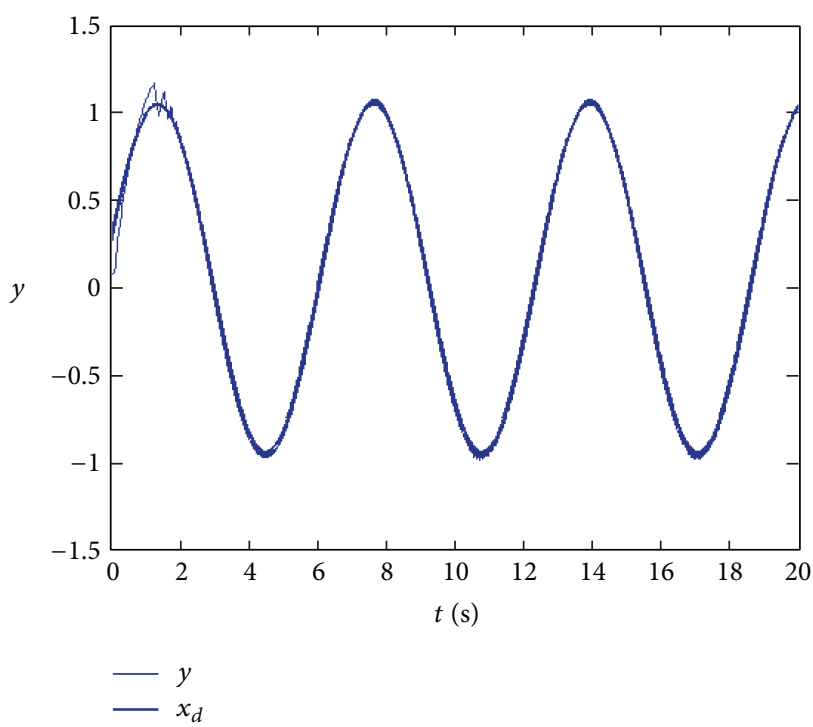

(b) Time domain chart of $y$ after control

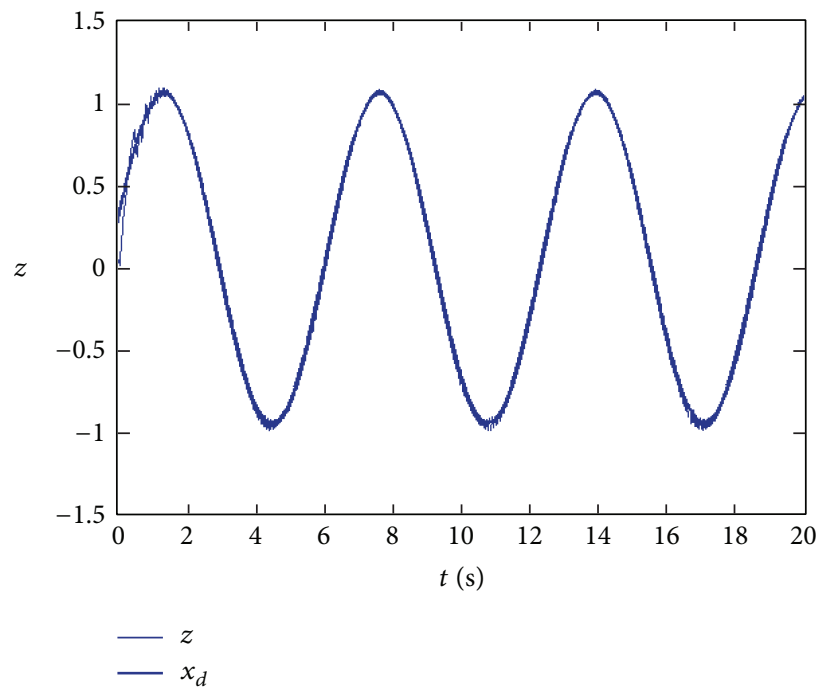

(c) Time domain chart of $z$ after control

Figure 14: Time domain charts of state variables after control.

about this chaotic system in terms of complexity, control, and synchronization, which deserve further investigation.

\section{Conflict of Interests}

The authors have declared that no conflict of interests exists.

\section{Acknowledgments}

The authors express their gratitude to the referee for valuable comments on the first version of the paper. The authors also gratefully acknowledge support from the National Natural Science Foundation (no. 11161027 and no. 61364001), Key Project of Chinese Ministry of Education (no. 212180), the Fundamental Research Funds for the Universities of Gansu
Province (no. 620023), and the Natural Science Foundation of Gansu Province (no. 1010RJZA067), Government of China.

\section{References}

[1] E. N. Lorenz, "Deterministic nonperiodic flow," Journal of the Atmospheric Sciences, vol. 20, pp. 130-141, 1963.

[2] G. Chen and T. Ueta, "Yet another chaotic attractor," International Journal of Bifurcation and Chaos, vol. 9, no. 7, pp. 14651466, 1999.

[3] J. Lü and G. Chen, "A new chaotic attractor coined," International Journal of Bifurcation and Chaos in Applied Sciences and Engineering, vol. 12, no. 3, pp. 659-661, 2002.

[4] Y.-D. Chu, X.-F. Li, J.-G. Zhang, and Y.-X. Chang, "Nonlinear dynamics analysis of a new autonomous chaotic system," Journal of Zhejiang University SCIENCE A, vol. 8, no. 9, pp. 14081413, 2007. 
[5] F. M. Amaral and L. F. C. Alberto, “Type-zero saddle-node bifurcations and stability region estimation of nonlinear autonomous dynamical systems," International Journal of Bifurcation and Chaos, vol. 22, no. 1, Article ID 1250020, 16 pages, 2012.

[6] J. Hai-Bo, L. Tao, Z. Xiao-Liang, and Z. Li-Ping, "Bifurcation analysis of the logistic map via two periodic impulsive forces," Chinese Physics B, vol. 23, Article ID 010501, 7 pages, 2014.

[7] D. Rezgui and M. H. Lowenberg, "Continuation nd bifurcation analysis in helicopter aeroelastic stability problems," Journal of Guidance, Control, and Dynamics, vol. 37, no. 3, pp. 889-897, 2014.

[8] M. M. El-Dessoky, M. T. Yassen, E. Saleh, and E. S. Aly, "Existence of heteroclinic and homoclinic orbits in two different chaotic dynamical systems," Applied Mathematics and Computation, vol. 218, no. 24, pp. 11859-11870, 2012.

[9] X. Chen, W. Huang, V. G. Romanovski, and W. Zhang, "Linearizability and local bifurcation of critical periods in a cubic Kolmogorov system," Journal of Computational and Applied Mathematics, vol. 245, pp. 86-96, 2013.

[10] B. W. Kooi, M. Aguiar, and N. Stollenwerk, "Bifurcation analysis of a family of multi-strain epidemiology models," Journal of Computational and Applied Mathematics, vol. 252, pp. 148-158, 2013.

[11] Y. Guo, W. Jiang, and B. Niu, "Bifurcation analysis in the control of chaos by extended delay feedback," Journal of the Franklin Institute: Engineering and Applied Mathematics, vol. 350, no. 1, pp. 155-170, 2013.

[12] W. Xu, J. Cao, and M. Xiao, "Bifurcation analysis and control in exponential RED algorithm," Neurocomputing, vol. 129, pp. 232-245, 2014.

[13] J. Páez Chávez, E. Pavlovskaia, and M. Wiercigroch, "Bifurcation analysis of a piecewise-linear impact oscillator with drift," Nonlinear Dynamics, vol. 77, no. 1-2, pp. 213-227, 2014.

[14] S. Li, Q. Wu, and Z. Zhang, "Bifurcation and chaos analysis of multistage planetary gear train,” Nonlinear Dynamics, vol. 75, no. 1-2, pp. 217-233, 2014.

[15] L. F. Mello, M. Messias, and D. C. Braga, "Bifurcation analysis of a new Lorenz-like chaotic system," Chaos, Solitons \& Fractals, vol. 37, no. 4, pp. 1244-1255, 2008.

[16] J. Sotomayor, L. F. Mello, and D. D. C. Braga, "Bifurcation analysis of the Watt governor system," Computational and Applied Mathematics, vol. 26, no. 1, pp. 19-44, 2007.

[17] Y. A. Kuznetsov, Elements of Applied Bifurcation Theory, vol. 112 of Applied Mathematical Sciences, Springer, New York, NY, USA, 3rd edition, 2004.

[18] F. S. Dias, L. F. Mello, and J. Zhang, "Nonlinear analysis in a Lorenz-like system," Nonlinear Analysis: Real World Applications, vol. 11, no. 5, pp. 3491-3500, 2010.

[19] X. Zhang, H. Zhu, and H. Yao, "Analysis of a new threedimensional chaotic system," Nonlinear Dynamics, vol. 67, no. 1, pp. 335-343, 2012.

[20] J.-D. Wang, T.-L. Lee, and Y.-T. Juang, "New methods to design an integral variable structure controller," IEEE Transactions on Automatic Control, vol. 41, no. 1, pp. 140-143, 1996.

[21] J.-H. Lee, P. E. Allaire, G. Tao, and X. Zhang, "Integral slidingmode control of a magnetically suspended balance beam: analysis, simulation, and experiment," IEEE/ASME Transactions on Mechatronics, vol. 6, no. 3, pp. 338-346, 2001.

[22] C. Di-Yi, L. Yu-Xiao, M. Xiao-Yi, and Z. Run-Fan, "Nochattering sliding mode control in a class of fractional-order chaotic systems," Chinese Physics B, vol. 20, no. 12, Article ID 120506, 9 pages, 2011.
[23] D. Y. Chen, T. Shen, and X. Y. Ma, "Sliding mode control of chaotic vibrations of spinning disks with uncertain parameter under bounded disturbance," Acta Physica Sinica, vol. 60, no. 5, Article ID 050505, 8 pages, 2011.

[24] D. Chen, P. Yang, X. Ma, and Z. Sun, "Chaos of hydro-turbine governing system and its control," Proceedings of the Chinese Society of Electrical Engineering, vol. 31, no. 14, pp. 113-120, 2011.

[25] Z. Z. Gao, "A new chaotic system," Journal of Hunan University of Arts and Science, vol. 23, pp. 34-47, 2011. 


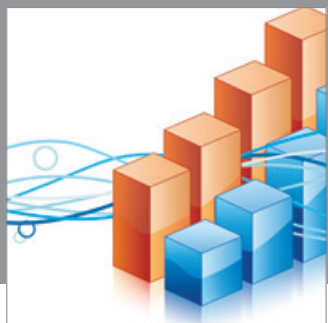

Advances in

Operations Research

mansans

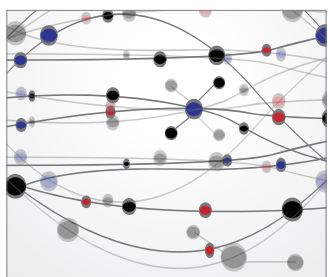

The Scientific World Journal
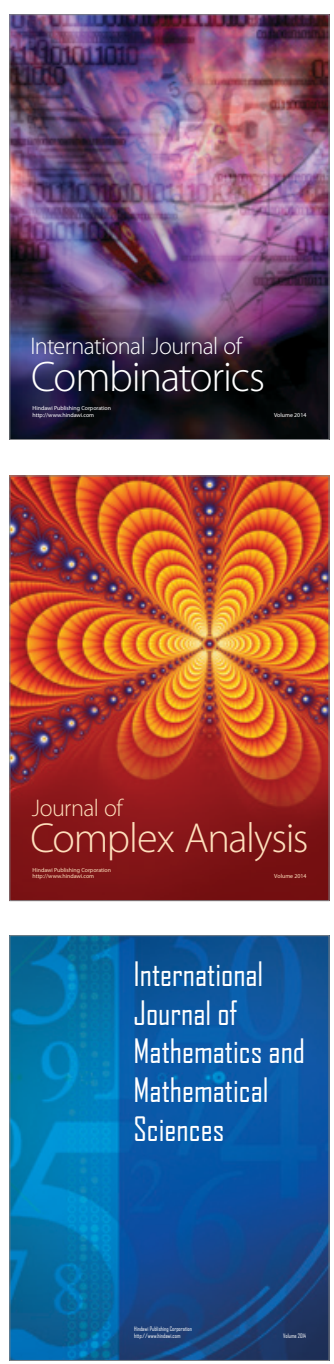
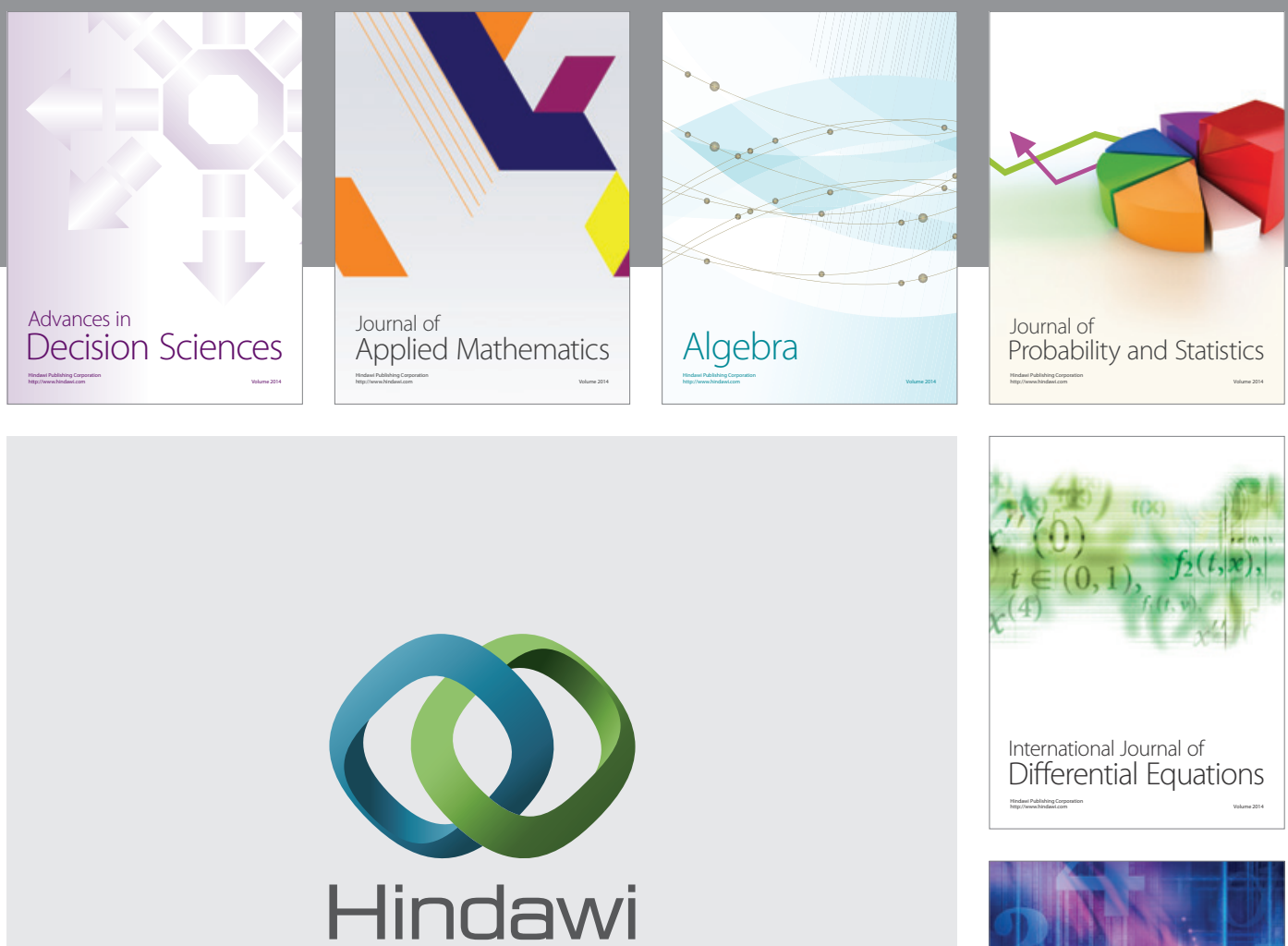

Submit your manuscripts at http://www.hindawi.com
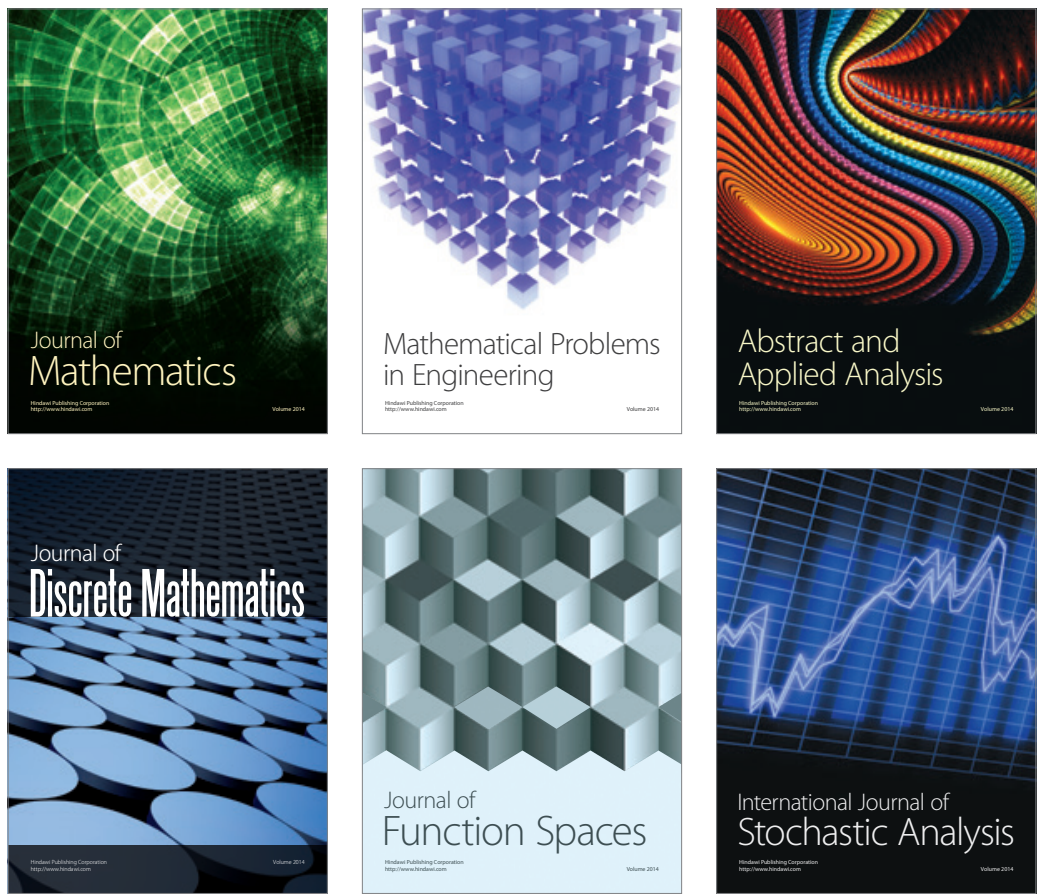

Journal of

Function Spaces

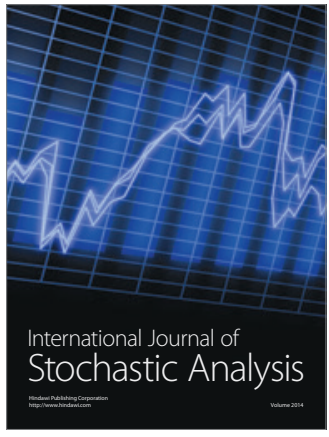

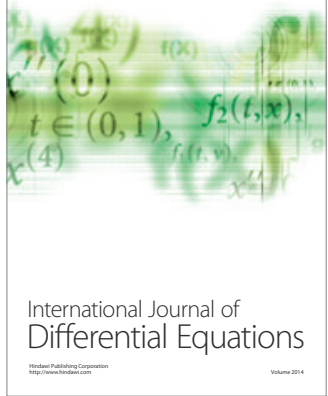
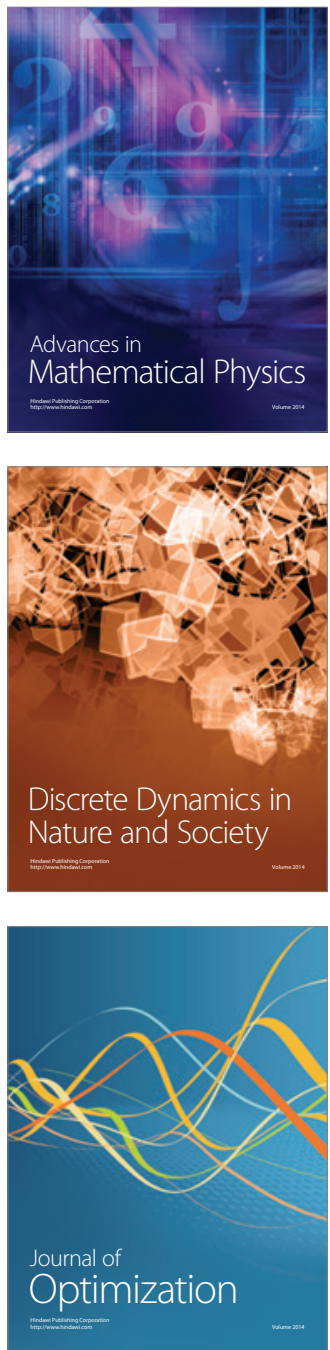